Boundary-Layer Meteor. manuscript No.

(will be inserted by the editor)

\title{
Towards reconciling the large-scale structure of turbulent boundary layers in the atmosphere and laboratory
}

\author{
Nicholas Hutchins - Kapil Chauhan • \\ Ivan Marusic • Jason Monty • Joseph \\ Klewicki
}

Received: date / Accepted: date

\begin{abstract}
A collaborative experimental effort employing the minimally perturbed atmospheric surface-layer flow over the salt playa of western Utah has enabled us to map coherence in turbulent boundary layers at very high Reynolds numbers, $R e_{\tau} \sim \mathcal{O}\left(10^{6}\right)$. It is found that the large-scale coherence noted in the logarithmic region of laboratoryscale boundary layers are also present in the very high Reynolds number atmospheric surface layer. In the atmospheric surface layer these features tend to scale on outer variables (approaching the kilometre scale in the streamwise direction for the present study). The mean statistics and two-point correlation map show that the surface layer under neutrally buoyant conditions behaves similarly to the canonical boundary layer. Linear stochastic estimation of the three-dimensional correlation map indicates that the low momentum fluid in the streamwise direction is accompanied by counter-rotating roll modes across the span of the flow. Instantaneous flow fields confirm the inferences made from the linear stochastic estimations. It is further shown that vortical structures aligned in the streamwise direction are present in the surface layer, and bear attributes that resemble the hairpin vortex features found in laboratory flows. Ramp-like high shear zones that contribute significantly to the Reynolds shear-stress are also present in the atmospheric surface layer in a form nearly identical to that found in laboratory flows. Overall, the present findings serve to draw useful connections between the vast number of observations made in the laboratory and in the atmosphere.
\end{abstract}

Nicholas Hutchins* ${ }^{*}$ Kapil Chauhan · Ivan Marusic · Jason Monty · Joseph Klewicki

Department of Mechanical Engineering, The University of Melbourne, Parkville 3010, Victoria, Australia

*E-mail: nhu@unimelb.edu.au

Joseph Klewicki

University of New Hampshire, Kingsbury Hall - W101, 33 Academic Way, Durham, NH 03824, USA 
Keywords Large-scale coherence $\cdot$ Neutral surface-layer $\cdot$ Turbulent boundary layer

\section{Introduction}

Turbulent boundary-layer dynamics affect a broad range of scientific and technological pursuits. As such, the approaches to investigating and describing boundary-layer phenomena vary between disciplines according to the needs of the given line of inquiry. Primary factors that colour the particular emphases include the Reynolds number regime, and whether the dynamics of the basic shear flow are influenced by other forces or boundary conditions, e.g. thermal stratification, pressure gradients or surface roughness effects. For this particular study, we attempt to draw links or parallels between two disciplines; studies of the atmospheric surface layer (ASL, defined as the lower part of the planetary boundary layer where the velocity profile is approximately logarithmic), and laboratory studies of turbulent boundary layers. Under certain conditions the physics between these two flows, both of which are wall-bounded external shear flows, should be identical. However, differences in approach, emphases and nomenclature between the engineering and meteorological research communities can often obscure such similarities.

Atmospheric surface-layer flows are invariably at high Reynolds number $\left(R e_{\tau}=\right.$ $\delta U_{\tau} / \nu=\mathcal{O}\left(10^{6}\right)$, where $\delta$ is the atmospheric surface-layer thickness, $U_{\tau}$ is the skinfriction velocity and $\nu$ is the kinematic viscosity), and thus its dynamics should be viewed within this context. Many wall-bounded turbulent flows in engineering applications also occur at very high Reynolds numbers (in this case $\delta$ is defined as the boundary-layer thickness, typically based on the distance from the surface where the mean velocity returns to some proportion of the freestream). In contrast, the vast majority of laboratory studies of boundary-layer turbulence have been conducted at low to moderate Reynolds numbers $\left[R e_{\tau}=\mathcal{O}\left(10^{3}-10^{4}\right)\right]$. Despite these different Reynolds number regimes, Reynolds number similarity tells us that the neutral ASL and the canonical flat plate boundary layer of the laboratory are precisely connected (e.g. if it were possible to compare the two at identical Reynolds numbers, both should be physically the same). The purpose of the present effort is to explore this connection at the detailed level of turbulence structure. Within the meteorological and engineering fields there have been a wealth of observations that inform us about boundary-layer structure, each coloured by the Reynolds number context described above. An important motivation for the present study is to identify the robust connections between these sets of observations, and thus advance understanding in both fields simultane-

ously. Prior to a presentation of current experiments, we therefore first review relevant studies from both the meteorological and the engineering literature. 


\subsection{Background}

The study of instantaneous structure within turbulent flows is important to identify mechanisms that, though pseudo-random, contribute to the similarity of time-averaged statistics in different flows. These mechanisms, once correctly identified, can be incorporated into wall- and sub-grid models to accurately compute industrial and atmospheric flows. As an example, the streamwise shift $\Delta s$ in the shifted Schumann-Grötzbach model that estimates the instantaneous surface shear-stress boundary condition (Piomelli et al. 1989) represents the average near-wall inclination angle of instantaneous turbulent structures. The term coherent structures is widely used to denote features in the flow that persist in space and time. For wall-bounded flows the existence of hairpin-shaped vortices, streaks, longitudinal rolls, burst and sweep events have all been identified and studied as coherent events. The nomenclature used here is often confusing and inconsistent between disciplines. As an example, it will be seen below, that the term 'streaks' has a very different meaning in the ASL literature than in the literature pertaining to laboratory turbulent boundary layers. A thorough reading on coherent structures in laboratory flows can be found in the reviews of Robinson (1991), Panton (2001), Klewicki (2010), Marusic et al. (2010b) and Smits et al. (2011). Here we limit our discussion to the structures seen in a zero-pressure-gradient turbulent boundary layer in the laboratory and the neutrally stratified ASL.

\subsubsection{Near-wall structure}

In this section, we discuss coherent events that reside within the first 100 wall units from the surface $\left(z^{+} \lesssim 100\right.$, where $z$ is the wall-normal distance and $\left.z^{+}=z U_{\tau} / \nu\right)$. For laboratory based flows, this would typically define an upper limit within the range $z<1-5 \mathrm{~mm}$ (where $z$ is the wall-normal ordinate) and for the ASL, this typically means within the first $5 \mathrm{~mm}$ or so from the wall. In the case of high Reynolds number engineering applications (such as the turbulent boundary layer forming over the fuselage of an aircraft or hull of a ship) this would translate to the first $0.5 \mathrm{~mm}$ from the wall. Coherent motions within this region are dominated by the near-wall cycle of streaks and quasi-streamwise vortices. Observed originally as a streakiness in the velocity field close to the wall (Kline et al. 1967; Robinson 1991), the near-wall structure has been extensively studied (more than any other coherent event in the turbulent boundary layer). These streaks consist of elongated (in the streamwise direction) spanwise alternating regions of high- and low-speed streamwise velocity fluctuations. Quasi-streamwise vor-

tices, approximately centred at $z^{+}=15$ (close to the near-wall peak in the variance of streamwise velocity fluctuations), accompany the streaks. Laboratory observations 
over a range of Reynolds numbers (Kline et al. 1967; Smith and Metzler 1983; Robinson 1991) have confirmed that the spanwise spacing and length of the near-wall streaks scale with the viscous length scale (average spanwise spacing of $100 \nu / U_{\tau}$ and streamwise length in the range $400-1000 \nu / U_{\tau}$ ). It should be noted that, for low Reynolds number laboratory flows or direct numerical simulations (DNS), the length of these near-wall events can be a significant portion of the outer length scale. However, for the ASL, such events are very small in comparison to $\delta$ (the typical length of the near-wall streak is $\left.\mathcal{O}\left(10^{-3}\right) \delta\right)$. It should also be noted that the near-wall structure will only exist over smooth or moderately (transitionally) rough surfaces. In the fully rough regime (roughness height $k \gtrsim 5 \mathrm{~mm}$ for the ASL), the near-wall dynamics are likely to be fully driven by the roughness structure and thus very different to smooth wall flow (Jiménez 2004). Despite these challenges, Klewicki et al. (1995) have confirmed the presence of streaks in the smooth-wall atmospheric surface layer (by ejecting theatrical fog from the ground), finding them similar in scale and form to those observed in laboratory flows (same characteristic spanwise spacing and length scale). In early visualization experiments (for example Kline et al. 1967), the streaks were often observed to undergo an oscillation and break-up referred to as 'bursting' or the 'burst cycle'. Inflows of high-speed fluid towards the wall associated with this cycle and ejections of low-speed fluid away from the wall were respectively termed 'sweeps' and 'ejections'. Subsequent studies have suggested a self-sustaining near-wall cycle of break-up and regeneration in which the streaks and vortices mutually self-sustain without the need of external influences or triggers (Jiménez and Pinelli 1999; Schoppa and Hussain 2002). In general, it is worth noting that the majority of near-wall cycle studies have been conducted at low Reynolds numbers, where the separation of scales and the influence of larger scales is by definition limited. It must be highlighted that the term 'streaks' as applied to the near-wall cycle refers to a very different scale and class of structure than the 'streaks' commonly referred to in the ASL literature (Boppe and Neu 1995; Wilczak and Tillman 1980; Moeng and Sullivan 1994; Lin et al. 1996; Drobinski and Foster 2003; Lu and Porté-Agel 2010). In a further complication, as analysis of coherent structures has progressed into the logarithmic and outer regions, the terms 'sweep' and 'ejection' have more recently been applied to Q2 and Q4 Reynolds shear-stress events associated with larger-scale structures located further from the wall. In general, caution must be exercised, due to the confusing inconsistencies in nomenclature between fields.

\subsubsection{The logarithmic region / outer region}

The logarithmic region is approximately defined as the region $100<z^{+}<0.15 R e_{\tau}$ for laboratory turbulent boundary layers. Owing to these limits, the logarithmic region 
is typically absent, or of minimal extent, for many low Reynolds number laboratory studies (if $R e_{\tau} \lesssim 700$, the region $100<z^{+}<0.15 R e_{\tau}$ does not exist). In contrast, for the ASL, the logarithmic region occupies almost the entire layer typically from a few millimetres above the surface up to $\mathcal{O}(100 \mathrm{~m})$. Again, this highlights the importance of accounting for Reynolds number effects. The structure of the ASL is dominated by logarithmic region structures, whilst low Reynolds number turbulent boundary layers are dominated by the near-wall cycle. This leads to boundary layers that can outwardly exhibit seemingly disparate structural behaviour, yet are in reality just at widely opposing ends of the Reynolds number continuum.

In laboratory studies, the existence of much larger structures in the logarithmic and outer regions of the flow has long been indicated by single-point statistics. Premultiplied energy spectra of streamwise velocity fluctuations have peaks occurring at large streamwise wavelengths. The long tails in the autocorrelations lead to similar conclusions. Two-point correlation measurements (e.g. Kovasznay et al. 1970; Nakagawa and Nezu 1981; Mclean 1990; Wark et al. 1991) revealed the presence of large-scale elongated regions of streamwise momentum deficit with length scales that approximately scale on $\delta$. The advent of particle image velocimetry (PIV) and higher Reynolds number DNS studies, provided the first quantitative instantaneous views of the logarithmic region structure, kick-starting a renewed focus on these larger scales. One of the most compelling results from emerging PIV studies involved observations of hairpin vortex structures arranged into groups or 'packets'. As early as the 1950s, hairpin vortices had been postulated as possible boundary-layer structures (Theodorsen 1952; Townsend 1956). The flow visualizations of Head and Bandyopadhyay (1981) suggested that the turbulent boundary layer was almost entirely populated by hairpin vortices. Laboratory-based PIV studies, initially from the Adrian group at Champagne-Urbana, provided evidence that these hairpin vortices are preferentially arranged into packets (Adrian et al. 2000b; Christensen and Adrian 2001; Tomkins and Adrian 2003). These packets form ramp-like structures with the hairpins at the downstream end of the packet extending further from the wall than the hairpins at the upstream end. Numerous other studies have investigated hairpin vortices and packets, both in laboratory (Ganapathisubramani et al. 2003, 2005; Hambleton et al. 2006; Wu and Christensen 2006; Dennis and Nickels 2011a, and others) and numerical investigations (Zhou et al. 1999; Wu and Moin 2009; Lee and Sung 2011, and others). Adrian (2007) provides an excellent review and overview of hairpin packet literature.

In the ASL, observations of hairpin packets are less prevalent. Carper and PortéAgel (2004) used data from an array of sonic anemometers to analyze conditionallyaveraged flow fields in the near-neutral ASL, finding hairpin vortex structures that are 
inclined downstream away from the wall at angles of about $16^{\circ}$. They noted sweep and ejection events associated with these structures. Flow visualization by Hommema and Adrian (2003) and particle image velocimetry by Morris et al. (2007) has provided some evidence for these events in the ASL. Though these studies were unable to prove the presence of hairpin vortices with the same clarity as observed in laboratory flows, they did show strong evidence of ramp-like shear layers and uniform momentum zones that are typical signatures of hairpin vortex packets (see Adrian et al. 2000b). A recent study by Herpin et al. (2010) has suggested that the vortex core radius scales approximately on the Kolmogorov length scale $\eta$ throughout the turbulent boundary layer, with an approximately constant radius of $8 \eta$ recorded across the layer. Hommema and Adrian (2003) employed a qualitative smoke visualization technique, which is not well suited to the resolution of individual small-scale features. For the PIV experiments of Morris et al. (2007), the expected core diameters can be estimated in the range $r^{+} \approx 30-42$ using the result of Herpin et al. (2010) and the logarithmic region estimate $\eta^{+}=\left(\kappa z^{+}\right)^{1 / 4}$ (where $\kappa$ is the von Kármán constant). This is smaller than the interrogation window used for the vector processing (and would thus exceed the spatial resolution of the system). In such instances, based on the results of Herpin et al. (2010) one would expect to resolve only the larger scale induced motions due to the vortices (or vorticity rich shear layers), rather than the actual vortex cores themselves. Regardless, the uniform momentum zones separated by inclined internal shear layers, observed by Morris et al. (2007), are strikingly reminiscent of the original hairpin packet observations of Adrian et al. (2000b), with reported differences between the high Reynolds number ASL structure and that of low Reynolds number laboratory flows restricted to the composition of the small-scale vortical motions within the shear layers. In other atmospheric studies, Phong-Anant et al. (1980) obtained temperature traces at several heights in the first $8 \mathrm{~m}$ of the atmospheric surface layer that indicated the presence of a ramp-like interface in the temperature profile, the inclination of which increased with height. Other experimental efforts have also found evidence of inclined structures in the ASL (Högström and Bergström 1996; Marusic and Heuer 2007; Guala et al. 2010). Vortical structures similar to hairpin vortices were found in a large-eddy simulation (LES) of the neutrally stratified planetary boundary layer (PBL) by Lin et al. (1996), but of quite different scale than those in a flat plate turbulent boundary layer.

\subsubsection{Very large-scale coherent motions}

In streamwise / spanwise planes within the logarithmic region, the packet structure manifests most strikingly as a pronounced large-scale stripiness in streamwise velocity 
fluctuations. The region within the hairpin packet is characterized by negative velocity fluctuations (and upwash). When this structure is sliced by streamwise / spanwise planes, highly elongated regions of negative streamwise velocity fluctuation are evident, flanked on either side by similarly elongated high-speed regions (e.g. Tomkins and Adrian 2003; Ganapathisubramani et al. 2003; Hambleton et al. 2006). These elongated regions often exceed the field of view of the PIV experiments. Energy spectra had long hinted that the structures in the logarithmic region could be very long indeed. Peaks in pre-multiplied energy spectra were known to occur at wavelengths of $\approx 6 \delta$ in the logarithmic region of turbulent boundary layers. Kim and Adrian (1999) had shown that for turbulent pipe flow the pre-multiplied spectra had a bi-modal appearance with peaks occurring at $3 \delta$ and $14-20 \delta$ that they termed large-scale motions (LSM) and very-large-scale motions (VLSM) respectively (see also Guala et al. 2006). In channel flow DNS, del Álamo et al. (2004) found similar large-scale contributions in energy spectra. Hutchins and Marusic (2007a) deployed spanwise rakes of hotwire probes in the logarithmic region of the turbulent boundary layer discovering meandering regions of highly elongated negative and positive velocity fluctuations occasionally exceeding $15 \delta$ in length. They termed these events 'superstructures'. Similar experiments performed in channel and pipe flows by Monty et al. (2007) have proven the existence of comparable events in internal boundary layers. In pipes and channels, these events appear to be on average slightly longer, and with slightly greater spanwise spacing and dimension than those found in the turbulent boundary layer. It has been shown that the superstructures/VLSM carry a large proportion of the Reynolds shear-stress (Ganapathisubramani et al. 2003; Guala et al. 2006). Additionally, Hutchins and Marusic $(2007 \mathrm{a}, \mathrm{b})$ have shown that the magnitude of the streamwise velocity fluctuations associated with the superstructure events increase in comparison to those of the nearwall cycle with increasing Reynolds number. (i.e. as Re increases, velocity fluctuations with wavelength equal to or greater than $\delta$ become increasingly energetic as compared to the smaller scale fluctuations arising from the near-wall cycle, see also Hutchins et al. 2009.) Thus large and very-large-scale events appear to increasingly dominate the turbulent structural landscape as the Reynolds number increases. In addition to the large-scale streak-like features associated with the superstructure/VLSM, there is also evidence that, in a mean sense at least, these features are associated with largescale counter-rotating roll modes of similar length (del Álamo et al. 2006; Marusic and Hutchins 2008). In the ASL, Inagaki and Kanda (2010) found elongated low momentum regions in the logarithmic layer of fully-rough atmospheric flow associated with active turbulence. 
Superstructures / VLSM have been observed to maintain a footprint all the way down to the surface, and thus they strongly influence the near-wall cycle. In addition to a clearly observed large-scale superposition (Abe et al 2004; Toh and Itano 2005; Hutchins and Marusic 2007a) it is noted that the large-scale events appear to steer the amplitude of the small-scale activity (Hutchins and Marusic 2007b; Marusic and Hutchins 2008; Mathis et al. 2009). This modulation has also been observed in LES of turbulent channel flow (Chung and McKeon 2010) and in the ASL (Guala et al. 2011). The notion of larger scales interacting with small-scales in this way is not new and has been extensively discussed in relation to shear flows (Bandyopadhyay and Hussain 1984) and the intermittency of fine scale turbulence (Sreenivasan 1985; Kholmyansky et al. 2007).

One outstanding question concerns whether the large-scale streaks observed in many ASL flows are related to the VLSM / superstructures recently observed in laboratory turbulent boundary layers. Roll modes and large-scale streak-like features are reported under many different circumstances and on many different scales for the planetary boundary layer. Young et al. (2002) give a good summary of these phenomena, categorizing them as shear-driven surface-layer streaks, shear and buoyancy driven surface-layer streaks, narrow and wide mixed-layer convective roll modes, and gravity waves induced by mixed-layer rolls. The latter three processes have vertical scales that far exceed the depth of the ASL, often spanning the majority of the mixed layer. Such convective processes are entirely absent from the majority of engineering / laboratory boundary-layer studies. It is important to recognize that the VLSMs / superstructures observed in flat plate turbulent boundary layers are solely a shear-driven phenomenon, occurring within the logarithmic region of the layer and as such should only be compared with the structure occurring within the neutrally buoyant ASL (i.e. the first category of events listed in Young et al. (2002)), where the turbulence is shear-driven and where one would expect an approximately logarithmic velocity profile. Large-scale streaks in the ASL have been observed in both experiments (Drobinski et al. 2004; Newsom et al. 2008) and numerical simulations (e.g. Moeng and Sullivan 1994; Khanna and Brasseur 1998; Carlotti 2002; Foster et al. 2006; Lu and Porté-Agel 2010). There are also numerous experimental observations of turbulent structure in the ASL that are consistent with an elongated structure (e.g. Boppe and Neu 1995; Wilczak and Tillman 1980). It is a source of some confusion that for some studies, these large-scale atmospheric observations have been compared to the behaviour of near-wall streaks observed in laboratory turbulent boundary layers. While the basic tenets of the large-scale events may seem qualitatively similar to near-wall streaks (i.e. anisotropy, accompanying vortical motions, sweeps and ejections etc.), they should be 
treated as different structures (this difference was noted by Carlotti 2002). For the typical ASL, large-scale 'streaks' are at a spatial scale that is $\mathcal{O}\left(10^{4}\right)$ times larger than the near-wall cycle. The near-wall cycle is either confined to the first few millimetres of the ASL, or entirely absent due to surface roughness effects. The large-scale elongated features observed in the ASL are logarithmic region events, presumably with different formation mechanisms, and as such should be compared with structures observed in the logarithmic region of laboratory flows.

In terms of origins, it has been suggested that superstructures/VLSM may form from concatenations of hairpin packets or bulges (Guala et al. 2006; Adrian 2007; Dennis and Nickels 2011b). Such models raise still more questions concerning the reason for such concatenation / self-organization, as well as wider questions concerning the origins of the bulges or packets. As an alternative, del Álamo et al. (2006) suggest that the large-scale streaks are passive wakes formed from attached vortical clusters that have grown up from the wall. In general, in discussing origins of wall-bounded turbulence, two distinct models have been proposed. Analysis of low Reynolds number flows has tended to lead to the proposal of 'bottom-up' models, in which it is hypothesized that structures grow upwards from the wall (from the near-wall cycle), to produce the largest scale motions. This model, while plausible at low $R e$, becomes increasingly difficult to countenance at high Reynolds number, where the separation in scales is very large (in the ASL, a true 'wall up' description requires something of length $\mathcal{O}(50 \mathrm{~mm})$ to grow into something $\mathcal{O}(\mathrm{km}))$. At high Reynolds numbers, 'top-down' models have been proposed (Hunt and Carlotti 2001; Hunt and Morrison 2001; Högström et al. 2002; Morrison 2007) in which large-scale outer-layer motions interact with the wall, creating elongated features in the logarithmic region and generating small-scale turbulence at the wall. These different interpretations raise questions about whether the mechanisms of turbulence generation and sustenance are different in low Reynolds number laboratory boundary layers than in the high Reynolds number ASL. At present, it would be wise to remain open to the possibility that both mechanisms co-exist (as suggested by Hunt and Morrison 2001) with the 'wall-up' mechanism perhaps prevalent at low $R e$ (due to the dominance of the near-wall cycle and the lack of scale separation) and 'top-down' mechanisms dominating at high Re (due to the increasing magnitude of the large-scale fluctuations and increasing scale-separation).

\subsection{Objectives}

From the above mentioned studies, the existence of coherence in both the ASL and in the laboratory turbulent boundary layer cannot be denied, although the commonality is often questioned. Documented inconsistences can stem from numerous factors in- 
cluding differences in Reynolds number, nomenclature, different focus / emphases, and the existence of additional convective effects. However, it is also generally the case that experiments in the atmosphere are inherently more challenging and can lack the resolution and confidence level typical of laboratory-based measurements. Keeping such challenges in mind the objectives of this paper can be listed as:

1. To examine the presence of very large-scale coherence in the atmospheric surface layer (ASL). This is achieved through calculating two-point correlation statistics and conditional-averaging. The presence of features suggested by these statistical techniques are verified in the instantaneous flow fields.

2. To compare the coherence in the laboratory and in the atmosphere using the same benchmark. For this we consider the zero-pressure-gradient turbulent boundary layer in the laboratory and neutrally stratified ASL flow over unusually smooth and flat terrain. Further, it is ensured that the same primary quantities are available for comparison in an equivalent three-dimensional measurement domain. Such a comparison goes beyond the conventional single-point mean and turbulence statistics results typically available from both flows (Klewicki et al. 1995; Metzger and Klewicki 2001; Marusic and Kunkel 2003).

3. To develop a unified understanding of structures that are common to laboratory and atmospheric flows; i.e. structures that are shear generated only. Both atmospheric and laboratory boundary-layer research communities have produced significant work in the fast emerging field of coherent structures. The present effort is an attempt to bridge gaps in nomenclature and approach and develop an integrated approach to simultaneously view structures in the ASL and laboratory turbulent boundary layer from the same perspective. The identified similarities or differences in the kinematics can then permit the extension of structure-based scaling ideas or predictions to very high Reynolds number; e.g., for wall-modelling or large-eddy simulations.

The outline of the paper is as follows: Sec. 2 describes the experimental set-up in the surface-layer measurements; Sec. 3 outlines the pre-processing and data selection methods associated with identifying a suitable subset of data, and Sec. 4 provides results that establish the equivalency of the chosen hour of neutral ASL data with laboratory zero-pressure-gradient turbulent boundary layers through mean statistics. Sec. 5 looks at large-scale features, through two-point correlation and linear stochastic estimates in three-dimensional space (Sec. 5.1) and instantaneous velocity field realizations (Sec. 5.2) that compliment the inferences made in Sec. 5.1. Sec. 6 looks at inclined vortical motions, again through two-point correlation (Sec. 6.1) and instantaneous velocity field realizations (Sec. 6.2). Conclusions are presented in Sec. 7. 

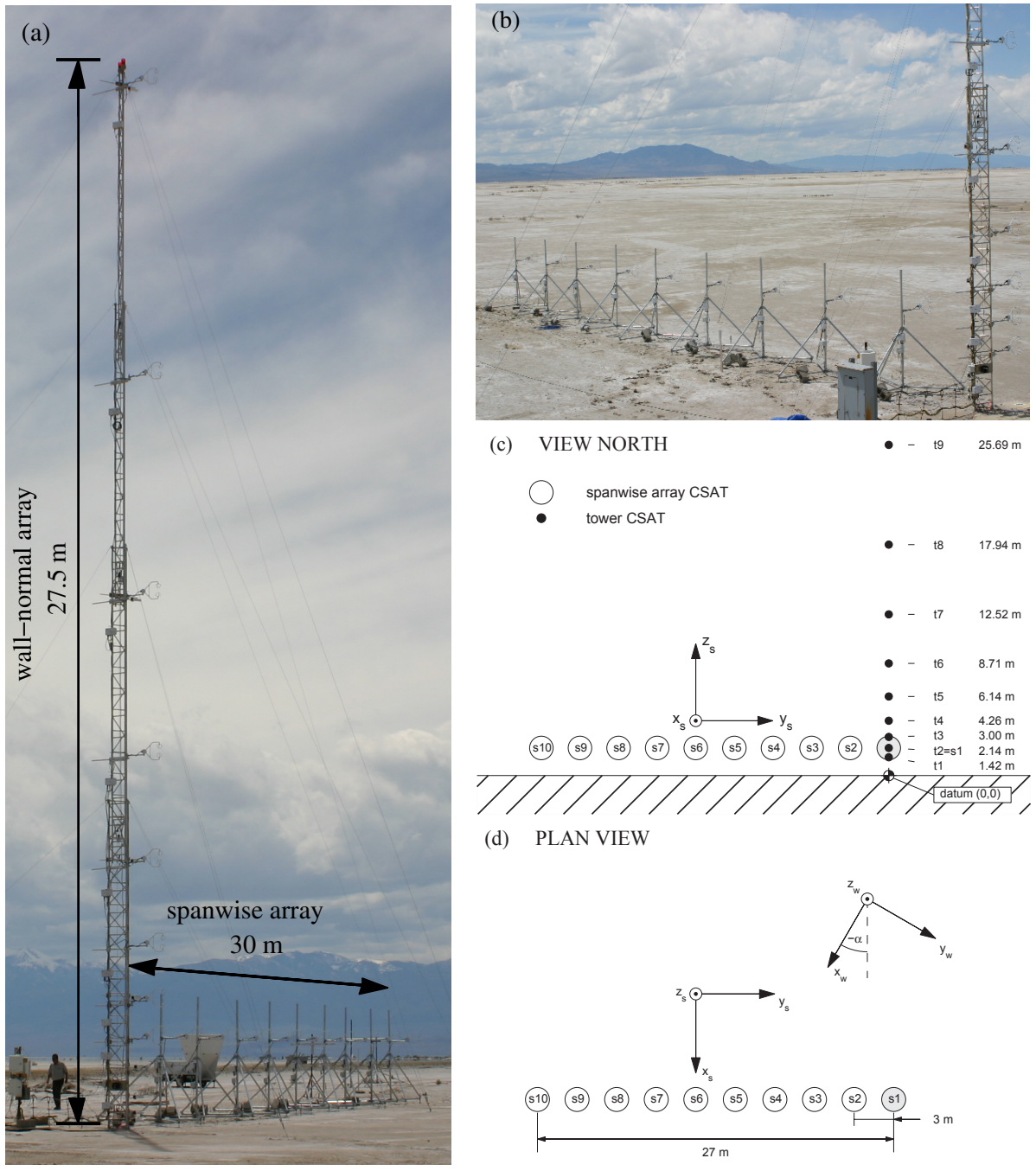

(d) PLAN VIEW

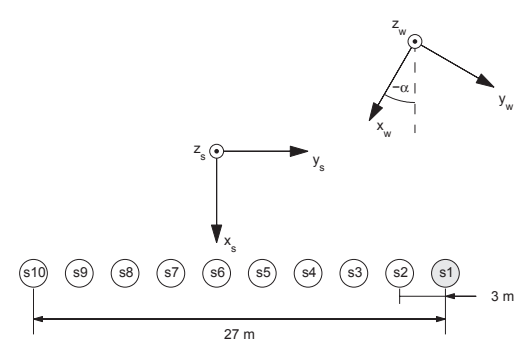

Fig. 1 (a) and (b) Two views of the measurement array installed at the SLTEST site (photographer D. Storwold). (c) north and (d) plan views of the sonic anemometer array.

\section{Facility}

The measurements were made at the Surface Layer Turbulence and Environmental Science Test (SLTEST) facility in the western salt flats of Utah over the period of 26 May 2005 to 4 June 2005. The measurement domain consists of a spanwise and wall-normal array of three-dimensional (3D) sonic anemometers (Campbell Scientific CSAT3) arranged in an ' $L$ ' shaped formation. The anemometers measure all three components of velocity along with temperature at a sampled rate of $20 \mathrm{~Hz}$ (all 18 
anemometers are sampled simultaneously). The measurement volume, and hence spatial resolution, of the sonic anemometers (determined from path length) is $100 \mathrm{~mm}$ $\left(\approx 1000 \nu / U_{\tau}\right)$ in the wall-normal direction and $58 \mathrm{~mm}\left(\approx 600 \nu / U_{\tau}\right)$ in the wallparallel direction. Photographs of the array installed at the SLTEST site are included in Figs. 1a-1b. Figures 1c and 1d show north and plan schematic views of the measurement array. Two right-handed coordinate systems are defined based on the principle measurement axis of the anemometer $\left(x_{s}\right)$ and the mean wind direction $\left(x_{w}\right.$, where $\alpha$ defines the angle between $x_{s}$ and $x_{w}$ ). For both axis systems, $z$ is the wall-normal ordinate. The array was aligned such that the principle measurement axis of the sonic anemometers ran north-south, giving a spanwise direction running east-west (this was based on the assumption that winds would be predominantly northerly at this time of year). The spanwise array comprises 10 devices, each mounted on tripods $2.14 \mathrm{~m}$ above the desert floor and spaced $3 \mathrm{~m}$ apart in the spanwise direction $\left(y_{s}\right)$. The wall-normal array consists of a further nine sonics spaced logarithmically in the vertical direction (from $z=1.42$ to $25.69 \mathrm{~m}$ ). The prefixes $\mathrm{s}$ and $\mathrm{t}$ are used to designate measurement stations in the spanwise array and tower respectively. Note that the first sonic in the spanwise array is shared with the second on the tower $(\mathrm{s} 1=\mathrm{t} 2)$. Throughout the paper capitalized velocities (e.g. $U$ ) and overbar indicate time-averaged values, lower case $(u)$ denote fluctuating components (so $\overline{u u}$ denotes the variance of the fluctuating component). The subscripts $s$ and $w$ relate the velocities to a particular axis system as defined

in Fig. 1. From the large amount of data that were gathered, we solely focus on one hour of data for an ASL that was approximately neutrally stratified ${ }^{1}$. The stability of the surface layer is characterized by the Monin-Obukhov stability parameter $z / \zeta(\approx 0$ for neutral conditions), where $\zeta$ is the Obukhov length

$$
\frac{1}{\zeta}=-\frac{\kappa g(\overline{w \theta})_{0}}{\bar{\theta} U_{\tau}^{3}},
$$

and where $\kappa=0.41$ is the von Kármán constant, $g$ is the gravitational acceleration, $U_{\tau}=(-\overline{u w})_{0}^{1 / 2}$ is the friction velocity obtained at $z=2.14 \mathrm{~m},(\overline{w \theta})_{0}$ is the surface heat flux, and $\bar{\theta}$ is the mean temperature.

\section{Data Selection}

For these types of measurement, obtaining an acceptable dataset is very much weather dependent. Although the array was sampled continuously for nine days, much of the

\footnotetext{
1 As standard practice in the analysis of surface-layer data, fluctuations of periods less than about one hour are considered as turbulence and the slower fluctuations as part of the mean field (Wyngaard 1992).
} 
acquired data are unsuitable for comparison with existing canonical boundary-layer results for reasons outlined in the following sub-sections. The first stage is to select usable data and the following section details criteria on which this selection is performed.

\subsection{Stability}

A measure of the stability of the ASL can be obtained from the wall-normal heat flux $(\overline{w \theta})$, which is merely a measure of the correlation between wall-normal velocity $(w)$ and temperature fluctuations $(\theta)$. The variation of $\overline{w \theta} / \sigma_{w} \sigma_{\theta}\left(\sigma_{w}\right.$ and $\sigma_{\theta}$ are the standard deviations of $w$ and $\theta$ fluctuations, respectively) for the spanwise array ( $z=$ $2.14 \mathrm{~m}$ ) over a period of several days is shown by the black line in Fig. 2a. The ambient temperature fluctuation for the same period is shown in Fig 2b. During the hottest parts of the day, $\overline{w \theta}$ records large positive values. In these instances we can see clear evidence of higher turbulence intensities for both the temperature and velocity fluctuations, indicative of buoyancy augmented turbulence production $\left(\tilde{U}_{s}=U_{s}+u_{s}\right.$ and $\tilde{V}_{s}=$ $V_{s}+v_{s}$ are shown in Fig 2c). With the approach of evening, the temperature drops and the effects of buoyancy diminish and the heat flux reduces to values that are close to zero. It is not uncommon for the heat flux to become negative at these times, leading to negative buoyancy or a 'stable' surface layer, in which turbulent motions are attenuated by gravity. However, for the current experiments, the heat flux remained close to zero for long periods during the night (for approximately 10 hours from 2000 $\mathrm{hr}$ to $0600 \mathrm{hr}$ the following morning). During these times the buoyancy effects can be considered negligible with turbulence production due just to shear (the neutral boundary layer). Only in these instances can we consider the ASL to be analogous to a flat-plate canonical turbulent boundary layer (and thus suitable for comparison with the wealth of existing experimental laboratory and computational results).

\subsection{Flow Direction}

In order to minimize interference and data contamination from the anemometer arms, tripods and other supporting structure, it is preferable that the sonic anemometer points into the mean wind direction (i.e. $x_{s}$ and $x_{w}$ are the same). During set-up, all sonic anemometers are nominally aligned northwards, with the spanwise array laid out perpendicular to this (along an east-west line, see Fig. 1). Whilst previous experience of the site indicates that the flow is predominantly northerly, there are many instances

where this is not the case. We define an acceptance cone of $\pm 30^{\circ}$ from the $x$ axis of the anemometer $\left(x_{s}\right)$. As an example, Fig. 2c indicates that for much of 31 May 2005 


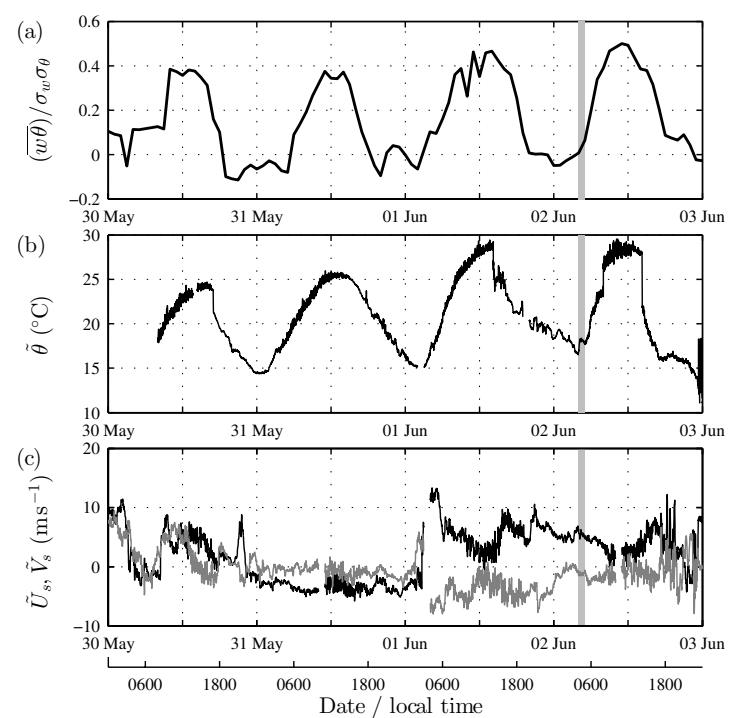

Fig. 2 Ambient conditions at the SLTEST site (a) the heat flux $\overline{w \theta}$; (b) instantaneous ambient temperature $\tilde{\theta}$; (c) streamwise (black) and spanwise (grey) total velocity fluctuation. All quantities are from the spanwise array at $z=2.14 \mathrm{~m}$.

the winds are predominantly southerly (the $\tilde{U}_{s}$ component is negative) and as such the entire day's data are discarded.

\subsection{Steady Winds}

The final requirement is that in the event of suitable neutrality and mean direction, the wind should be steady. Namely, an approximately constant streamwise velocity, without gusting or drastic changes in mean direction. In order to obtain converged statistics on the low wavenumber events in the turbulent boundary layer, these conditions should be maintained for a reasonable period of at least 30 minutes, which at $5 \mathrm{~ms}^{-1}$ equates to an advection length of $\mathcal{O}(100)$ boundary-layer thicknesses. These sifting criteria were met briefly during the early hours of 2 June 2005 (the shaded rectangle in Fig. 2 a - c mark these periods). Figure 3a shows in greater detail the streamwise and spanwise velocity trace for the hours 0400 to 0500. Based on $U_{s}$ and $V_{s}$ for this hour, the mean flow angle $\alpha \approx-12^{\circ}$. The stability parameter during this period is less than 0.1 (Obukhov length, $\zeta \approx 1000 \mathrm{~m}$ ) and hence the boundary layer can be considered neutral. It is still necessary to perform some additional data treatment and de-trending on the data, which is detailed in the following section. 


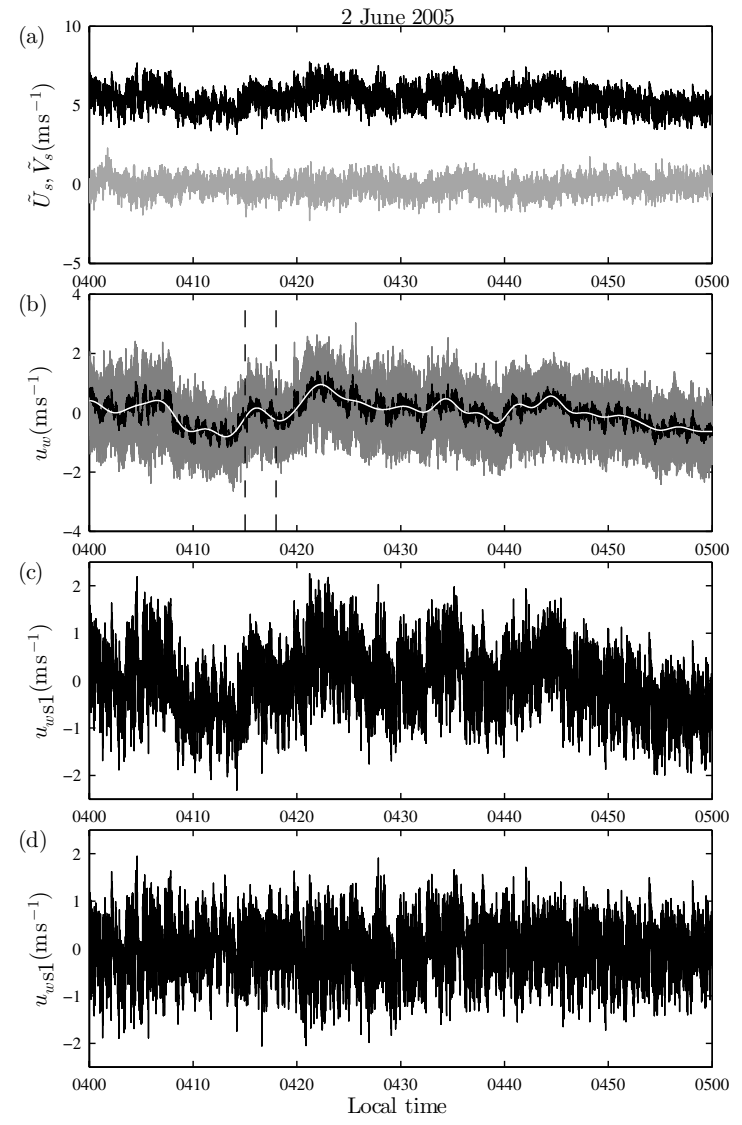

Fig. 3 (a) Streamwise (black) and spanwise (grey) instantaneous velocity time series for the selected hour sampled from $\mathrm{s} 1=\mathrm{t} 2$. (b) Streamwise fluctuating velocity signal across the entire array; Solid grey lines, $u_{w}$ for all 18 sonics; Solid black line, the mean of these signals; Solid white line, spectrally filtered average across the array $u_{L S}$; vertical dashed lines indicate the filter size (c) original $u_{w}$ signal on s1; (d) de-trended signal of s1.

\subsection{De-trending}

The raw velocity data for 0400 to 0500 on 2 June 2005 shown in Fig. 3a is relative to the measurement axis of the sonic anemometer. The first stage in data-treatment is to convert from this coordinate system to one based on the mean wind direction over the hour (these axis systems are defined in Fig. 1). A mean flow angle is established for the hour $\left(\alpha \approx-12^{\circ}\right)$ from which the following trigonometric conversion yields the true streamwise and spanwise velocity fluctuations

$$
\begin{aligned}
& u_{w}=u_{s} \cos (\alpha)+v_{s} \sin (\alpha), \\
& v_{w}=v_{s} \cos (\alpha)-u_{s} \sin (\alpha) .
\end{aligned}
$$


The primary aim of this analysis is to look for structures and correlations in the logarithmic region of a high Reynolds number turbulent boundary layer. Correlation estimates can be extremely sensitive to long-term (non-turbulence related) trends in the data. In this case such trends are caused by a varying freestream velocity (which is due to the natural variability of the ASL). Such a trend is clearly visible in the $\tilde{U}_{s}$ signal of Fig. 3a, where the turbulent fluctuations appear to be modulated by a long-term trend. Separating these two effects with simple filtering techniques carries the inherent risk that in removing long-term trends one also inadvertently filters out low wave-number boundary-layer motions. The largest-scale motions in a turbulent boundary scale on boundary-layer thickness (Mclean 1990; Wark et al. 1991; Hutchins and Marusic 2007a), and hence for the ASL (where $\delta$ is large) these will attain very large physical dimensions. Recent results of Hutchins and Marusic (2007a) seem to suggest that events of length $10-20 \delta$ are not uncommon in laboratory-scale turbulent boundary layers. As an example, if we assume a $\delta$ for the ASL of $60 \mathrm{~m}$, and a convection velocity of $5 \mathrm{~ms}^{-1}$, such motions could have advection times of $\mathcal{O}(2-4$ minutes $)$. Clearly such time-scales overlap with those we might typically associate with weather related phenomena. Fortunately, the current dataset has the advantage of simultaneous velocity measurements over a large spatial array. Since the largest-scale turbulence events tend to be rather more compact in the spanwise direction than the streamwise, we can make a broad assumption that any events registered across the entire domain are weather related, and subtract this large-scale trend from the raw signals to leave just the turbulent fluctuations ${ }^{2}$. Figures $3 \mathrm{~b}-3 \mathrm{~d}$ illustrate this process. The fluctuating velocity signals from all 18 sonics in the spanwise and wall-normal arrays are averaged together. The 18 original signals are plotted with grey lines on Fig. 3b, the average is shown in black. The broad trends noted in this average signal represent events registered across the entire measurement domain and as such are assumed to be due to weather phenomena. This averaged signal is high-pass filtered using spectral methods and a cut-off wavelength of $1 \mathrm{~km}$ (equivalent to $180 \mathrm{~s}$ ) to leave just the large-scale weather trend $u_{L S}$ (shown by the white line on Fig. $3 \mathrm{~b}$, where the filter size is also shown). Velocity data are de-trended by subtracting this large-scale trend from the raw fluctuating signal. As an example of this process, Fig. 3c \& 3d respectively show the uncorrected $u_{w}$ from s1 and the corresponding de-trended signal (the white line in plot b subtracted from the signal in plot c). The remainder of the analysis covered here

\footnotetext{
2 This assumption would be better justified if we had used a larger measurement array (covering a spanwise domain larger than the current $\delta / 2$ ). Alternatively, a single reference sonic located some distance (greater than the largest spanwise correlation length scale) to the east or west of the measurement domain (say $5 \delta$ ) would have enabled more confident optimal filtering of weather related phenomena (see Naguib et al. 1996).
} 

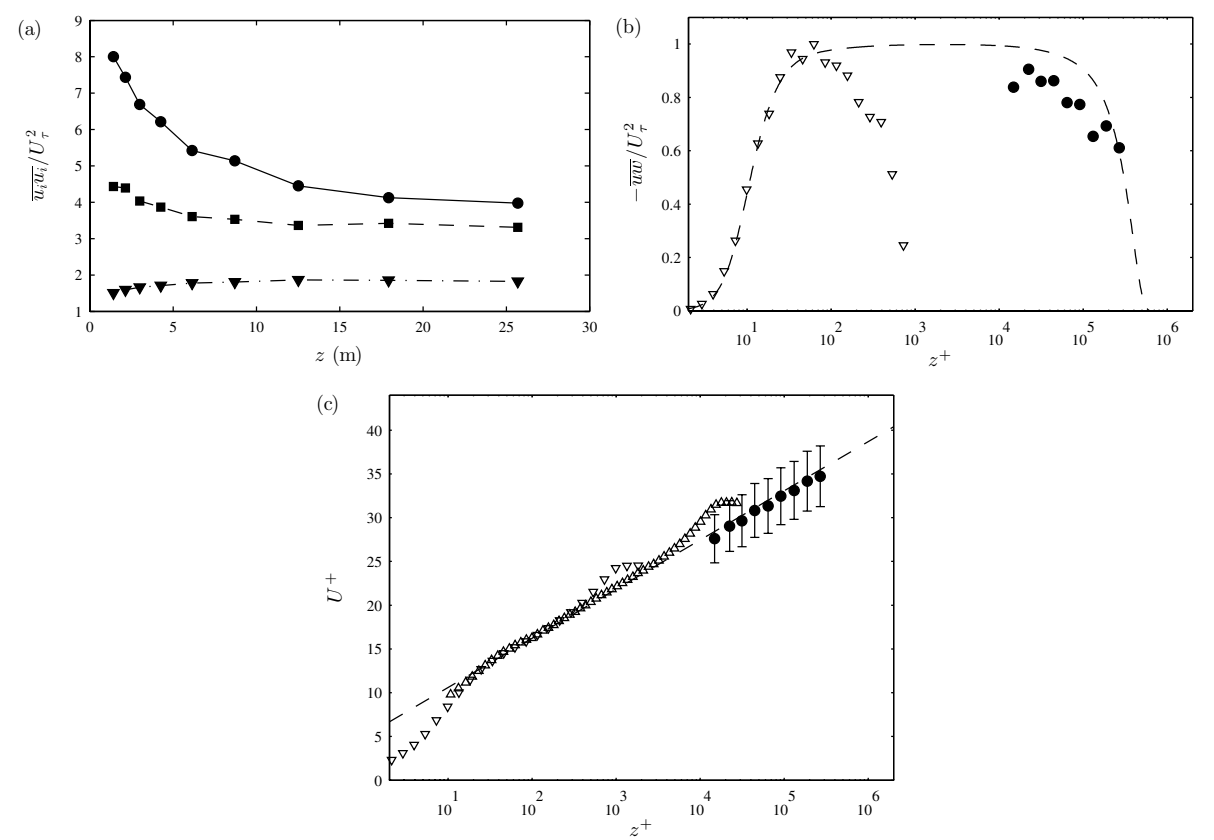

Fig. 4 Statistics from the tower array; (a) inner-scaled turbulence intensities from tower array.

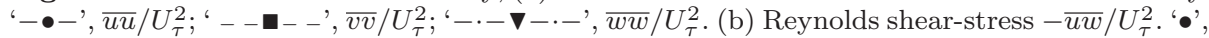
current SLTEST data, $\operatorname{Re}_{\tau} \sim \mathcal{O}\left(10^{6}\right) ; \nabla$, low Re laboratory data of DeGraaff and Eaton (2000), $R e_{\tau}=1350$; '- - -', similarity formulation from Chauhan (2007) (c) inner-scaled mean velocity profile. ' $\bullet$ ', current SLTEST data, $R e_{\tau} \sim \mathcal{O}\left(10^{6}\right)$; ' $\nabla$ ', data of DeGraaff and Eaton (2000), $R e_{\tau}=1350$; ' $\Delta$ ', data of Mathis et al. (2009), $R e_{\tau}=19030$; '- - '', $U^{+}=1 / 0.41 \ln \left(z^{+}\right)+5.0$

will deal exclusively with this de-trended data in the wind direction and the subscript ' $w$ ' is not used.

\section{Validation}

It is prudent to look at some basic mean statistics from the selected hour of data and check these against expected results from a canonical turbulent boundary layer. Figure 4a shows the turbulent intensities for the three velocity components, while the Reynolds shear-stress $-\overline{u w}$ is plotted in Fig. 4b. All quantities are normalized by the friction velocity $U_{\tau}$. In the absence of an independent measurement of wall shear, $U_{\tau}$ is estimated from the peak in the Reynolds shear-stress profile (such that the average $-\overline{u w} / U_{\tau}^{2}$ across the spanwise measurement array peaks at unity). The results of DeGraaff and Eaton (2000) would seem to suggest that estimating $U_{\tau}$ from peak $-\overline{u w}$ is a somewhat inaccurate method of determining wall shear (at least for lower Reynolds numbers) and as such the present estimate of $U_{\tau}$ should probably not be considered accurate beyond approximately $\pm 10 \%$. Regardless, the quantitative 
trends in the $u, v$ and $w$ variance look reasonable. Perhaps intuitively, the rising value of $\overline{w w}$ below $z \approx 12 \mathrm{~m}$ looks surprising. However, a closer examination of existing laboratory and experimental results (e.g. Spalart 1988; DeGraaff and Eaton 2000) reveals that this is expected up to approximately $0.2 \delta$. According to Högström et al. (2002), the increase of vertical velocity variance with height is caused by detached eddies of relatively large scale, that are being brought down from the upper surface layer. With an estimate of $U_{\tau}$, the variation of $-\overline{u w} / U_{\tau}^{2}$ for the surface layer is compared with the prediction of Chauhan (2007) and Nagib and Chauhan (2008) for a zeropressure-gradient turbulent boundary layer. Attempting this comparison requires us to first estimate the Reynolds number for the atmospheric measurements, which in turn requires an estimate of the ASL thickness $\delta$. Unfortunately $\delta$ was not directly measured for this particular measurement campaign, and consequently a value of $\delta$ must be estimated for consistency across a range of measured statistics. In this case $\delta$ is estimated for the atmospheric studies from comparisons of $\overline{u u}$ and $-\overline{u w}$ profiles (and also two-point correlation results) with laboratory data. The optimum consistency is returned for an estimate $\delta=60 \mathrm{~m}$, which yields a Reynolds number $R e_{\tau}=7.7 \times$ $10^{5}$. The theoretical prediction of Chauhan (2007) and Nagib and Chauhan (2008), based on a composite mean velocity profile, is formulated at this Reynolds number in Fig. 4b. Within expected experimental error, the measured data lies close to this predicted profile which validates that during this particular hour of data the ASL exhibits turbulence statistics that are as predicted from laboratory based studies of flat-plate turbulent boundary layers (based on an assumed $\delta=60 \mathrm{~m}$, this estimate is further verified for consistency with statistics in Figs. 5 and 6 ). The overall trend of $-\overline{u w}$ stress from the ASL measurements compared to the low Re data of DeGraaff and Eaton (2000) $\left(R e_{\tau}=1350\right)$ also indicates that it is appropriate to consider that the peak Reynolds stress occurs within the wall-normal span of the tower array. Figure 4c shows the boundary-layer profile non-dimensionalized, and plotted logarithmically. It is clear that the estimate of $U_{\tau}$ from the Reynolds shear-stress profile is in agreement with the Clauser chart. The ASL data agrees very well with the logarithmic law (where $\kappa=0.41$ and $A=5.0$ ) which would seem to indicate that the desert surface was hydrodynamically smooth. However, the included error bars (which show the supposed $\pm 10 \%$ error in the estimate of $U_{\tau}$ ) indicate that if the actual $U_{\tau}$ was $10 \%$ higher, the surface layer could be in the transitionally rough regime with an indicated equivalent sand-grain roughness height of $k_{s}^{+} \approx 21$, equating to $k_{s}=2 \mathrm{~mm}$ (close to the values measured in 2002 by Kunkel 2003). By way of comparison with lower Reynolds number data, Fig. 4c also includes boundary-layer profiles at $R e_{\tau}=1350$ (DeGraaff and Eaton 2000) and $R e_{\tau}=19030$ (Hutchins et al. 2009) from laboratory measurements. This 

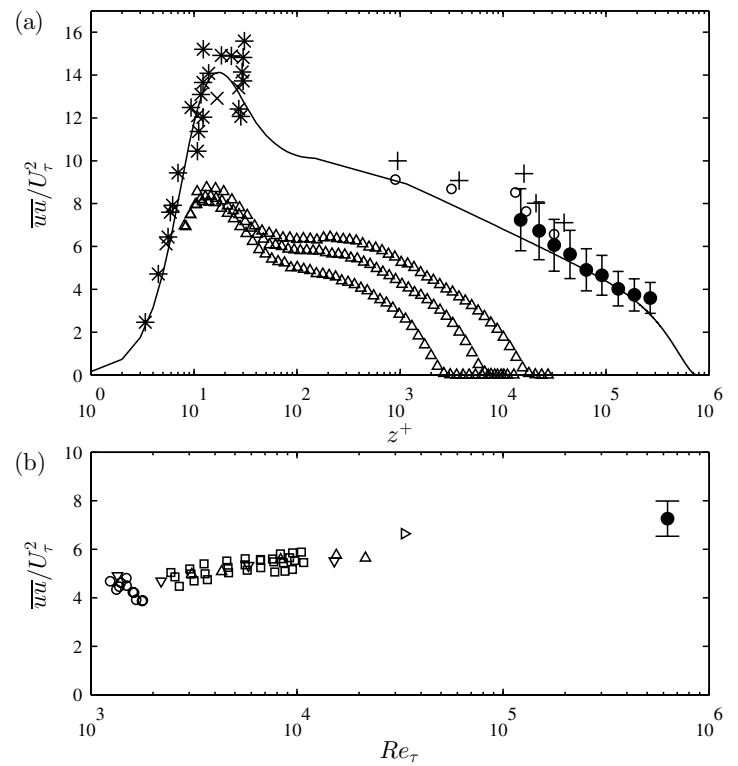

Fig. 5 Comparison of $\overline{u u} / U_{\tau}^{2}$ experimental data with the similarity formulation from Marusic and Kunkel (2003). (a) ' $\bullet$ ', current SLTEST data; symbols $\circ$ and + are data of Kunkel and Marusic $(2006)$ at $R e_{\tau} \approx 3.1 \times 10^{6}$ and $3.8 \times 10^{6}$, respectively. symbols $\times$ and * are data of Metzger et al. (2001) at $R e_{\tau} \approx 8.3 \times 10^{5} ; \Delta$, data of Hutchins et al. (2009), $R e_{\tau}=2800,7300$, and 19030. Line shows the similarity formulations proposed by Marusic and Kunkel (2003) calculated at $R e_{\tau}=7.7 \times 10^{5}$. (b) Variation of $\overline{u u} / U_{\tau}^{2}$ at $z / \delta=0.036$ with $R e_{\tau} ;$ ' $\bullet$ ', current SLTEST data from the spanwise array; ' 0 ' data of Kulandaivelu and Marusic (2010); ' $\square$ ', Österlund et al. (2000); ' $\Delta$ ', Hutchins et al. (2009); ' $\triangleright$ ', Knobloch and Fernholz (2002).

is a good graphical representation of the Reynolds number range over which we have analyzed the largest scale structures in turbulent boundary layers (almost three orders of magnitude).

There is a shortage of high Reynolds number data against which to compare the ASL results. However, Fig. 5a is a reproduction of the streamwise turbulent intensity data including previous measurements at the SLTEST site, laboratory data and the similarity formulation presented in Marusic and Kunkel (2003). The current data are shown with solid symbols, with the error bars representing the uncertainty in $U_{\tau}$. The Marusic and Kunkel (2003) formulation for $\overline{u u} / U_{\tau}^{2}$ based on the current Reynolds number is represented by the solid line. The reasonable agreement suggests that our measured ASL data agrees well with this theoretical formulation based on laboratory turbulent boundary layers (providing further validation of the estimate for $\delta$ ). The current data also agrees well with previous measurements at the SLTEST site. The variance measured from the tower compares well with the logarithmic region results of Kunkel and Marusic (2006) (shown by the plus signs and open circles). Figure 5b 
plots $\overline{u u} / U_{\tau}^{2}$ measured at $z / \delta=0.036$ from the spanwise array in comparison to several laboratory measurements at the same outer-scaled location. It is clearly evident that a wide Reynolds number gap exists between the laboratory data and the current ASL result. However, the surface-layer measurement agrees with the extrapolated trend from laboratory data. In general then, Figs. 4 and 5 show that within the quoted uncertainty for $U_{\tau}$ and assuming an estimate for the surface-layer thickness of $\delta=60 \mathrm{~m}$, the nearneutral ASL at the SLTEST site seems to be behaving like a canonical turbulent boundary layer during the selected hour of data.

\section{Evidence of very large-scale coherence}

5.1 Statistical evidence: Two-point correlations

Figure 6 shows a comparison of the outer-scaled two-point correlation coefficient of streamwise velocity fluctuation $\left(R_{u u}\right)$ for both existing laboratory data and the ASL results reported here (the laboratory turbulent boundary layer results are reproduced from Hutchins and Marusic 2007a). Plot (a) shows the correlations in the streamwise direction at $\Delta y=0$ (this is the autocorrelation). The streamwise ordinate in these figures is recovered using Taylor's hypothesis, taking a convection velocity based on the mean across the spanwise array ${ }^{3}$. Figure $6 \mathrm{~b}$ shows the spanwise variation of $R_{u u}$. The data shown in black are existing laboratory results for $z / \delta=0.05$ and for a Reynolds number range $1840<R e_{\tau}<19950$. These data were found by Hutchins and Marusic (2007a) to exhibit a good collapse in $R_{u u}$ when scaled using outer-variables. In attempting to present ASL data from SLTEST on these figures, we are faced with the problem that for these particular atmospheric measurements we do not have a precise value for $\delta$ (which is typically defined as the thickness of the ASL). However, the curves shown in red on plots (a) and (b) indicate that if we assume a value for $\delta$ of $60 \mathrm{~m}$ (which seems reasonable from Section 4), the two-point correlations collapse reasonably well with the existing laboratory results. This estimate of $\delta$ would actually yield a $z / \delta$ of 0.036 for the ASL spanwise array, which is not directly comparable with the laboratory data shown in Fig. 6 (for which $z / \delta=0.05$ ). However, a comparison of the $R_{u u}$ results from Hutchins and Marusic (2007a) for varying $z / \delta$ would suggest that there should be very little change between the curves at $z / \delta=0.036$ and 0.05 . It should also be cautioned that the advection lengths here (in terms of boundary-layer thicknesses) are very low for the ASL data. Even with one hour of stable data, the advection length is only approximately $330 \delta$ (assuming $\delta \approx 60 \mathrm{~m}$ ). By comparison, the laboratory velocity

\footnotetext{
3 Dennis and Nickels (2008) showed that Taylor's approximation is accurately applicable when the projection distances are small $(<6 \delta)$ and only the large scales are of interest.
} 

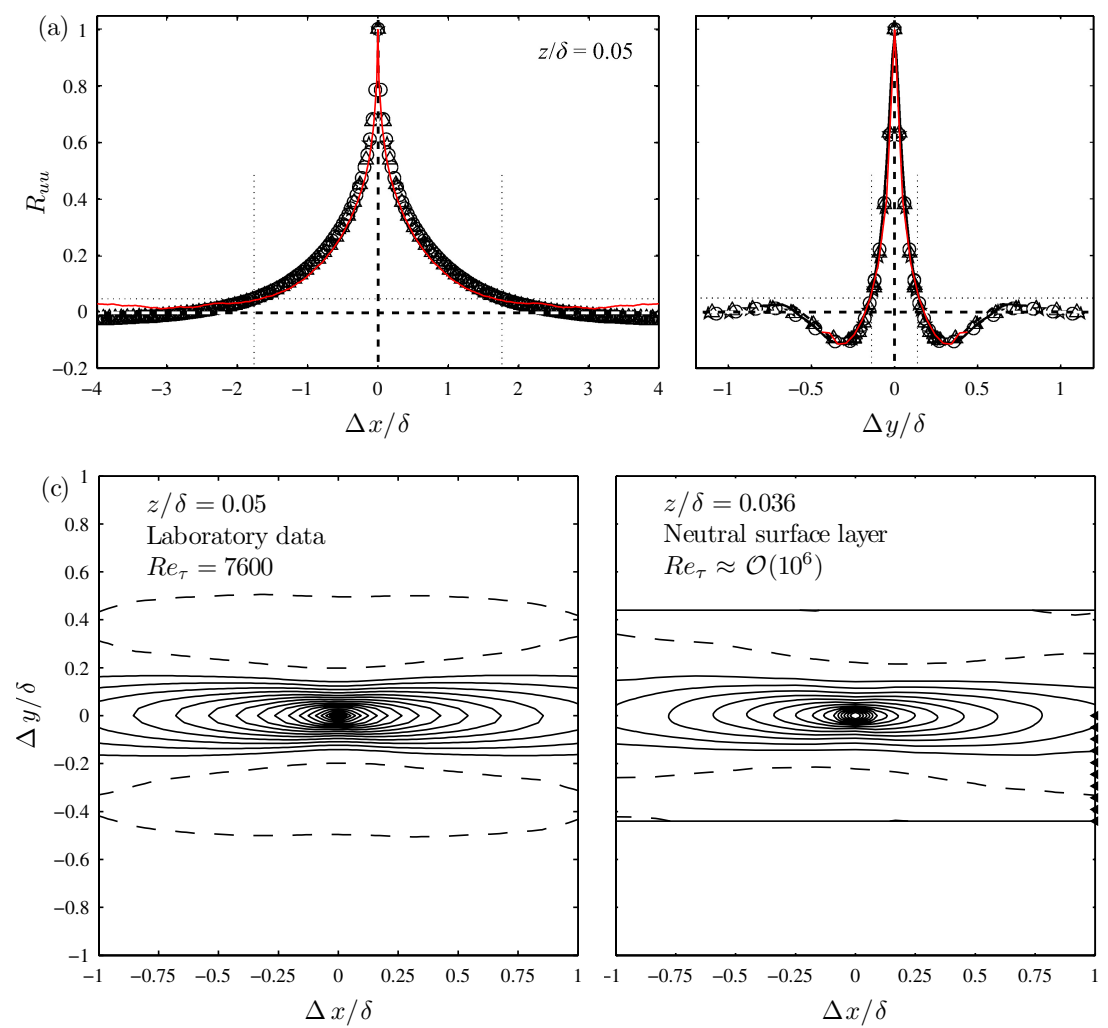

Fig. 6 Two-point correlations of the streamwise velocity fluctuation $R_{u u}$ calculated for laboratory and ASL data at $z_{r e f} / \delta \approx 0.05$. (a) streamwise (b) spanwise. Symbols show laboratory rake data (Hutchins and Marusic 2007a) (o) $R e_{\tau}=7600$; $(\triangle) R e_{\tau}=14400$; ( $) R e_{\tau}=19950$. Black lines show $45^{\circ}$ inclined plane PIV results (Hutchins et al. 2005) for (dashed) $R e_{\tau}=1840$; (solid) $R e_{\tau}=2800$. Solid red line shows the ASL data at $z_{r e f} / \delta \approx 0.036$. Plots (c) and (d) show iso-contours of $R_{u u}$ over a streamwise / spanwise domain for (c) the laboratory hot-wire rake data at $R e_{\tau}=7600$ and (d) the ASL spanwise rake data at $R e_{\tau} \approx \mathcal{O}\left(10^{6}\right)$. Contour levels for both plots are from $R_{u u}=-0.12$ to 0.96 in increments of 0.06 . Dashed lines show negative contours. The horizontal lines on (d) represent the maximum $\Delta y$ (the spanwise limit of the correlation data). Symbols ' $\varangle$ ' indicate the position of 10 sonics in the spanwise array.

signals for the $R e_{\tau}=19950$ data shown in Fig. 6 have advection lengths of over $37000 \delta$. This is a good reminder of the difficulties faced in obtaining converged statistics at the SLTEST facility (the same level of convergence for the ASL data would require more than 100 hrs of stationary data). Regardless, the collapse shown in Fig. 6 would seem to suggest that the largest-scale structures in the turbulent boundary layer scale on (something close to) boundary-layer thickness up to $R e_{\tau} \approx \mathcal{O}\left(10^{6}\right)$. The physical variation in scale represented by the $R_{u u}$ profiles in Fig. 6 is very large. As an example, in plot (b) the dimensional distance between the two negative peaks in the $R_{u u}$ profile (occurring at $\Delta y \approx \pm 0.3 \delta$ ) ranges from just $44 \mathrm{~mm}$ at $R e_{\tau}=1840$ (dashed line), 


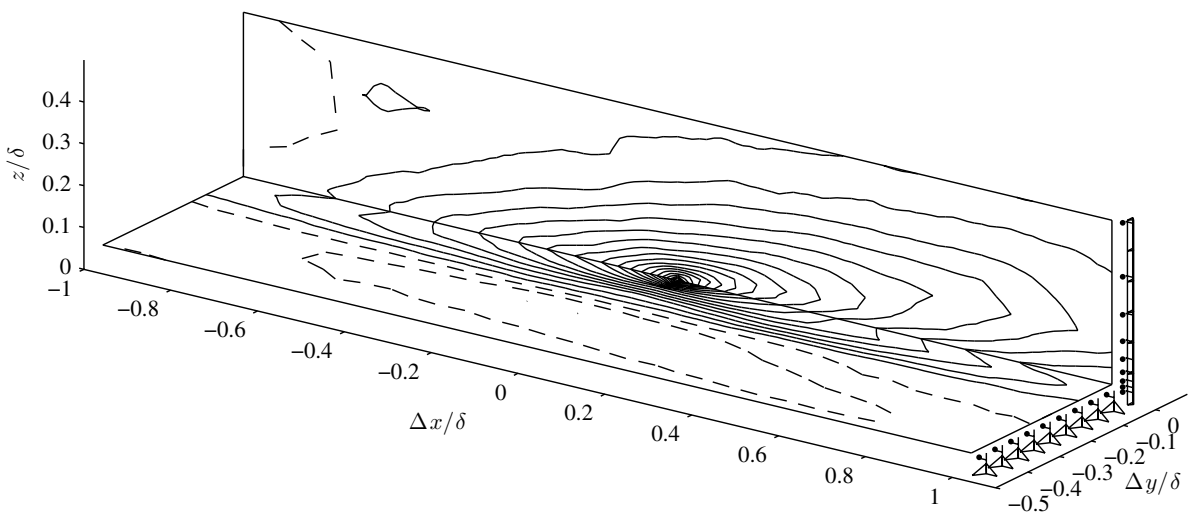

Fig. 7 Iso-contours of $R_{u u}$ for the ASL data for both the spanwise array and the tower. The condition point is at $\mathrm{s} 1(=\mathrm{t} 2)$. Contour levels are from $R_{u u}=-0.15$ to 0.95 in increments of 0.05 . Solid lines show positive contours and dashed lines show negative levels. Symbols ' $\bullet$ indicate the position of sonic anemometers.

through to approximately $20 \mathrm{~cm}$ at $14400<R e_{\tau}<19950$ (black symbols) all the way to $36 \mathrm{~m}$ for the atmospheric surface layer (red curves).

For further comparison, Fig. $6 \mathrm{c}$ and $6 \mathrm{~d}$ show the full streamwise/spanwise two-point correlation maps of $R_{u u}$ for both the laboratory hot-wire rake data at $R e_{\tau}=7600$ and the SLTEST data respectively. In both cases, a region of positive correlation, approximately $0.35 \delta$ wide and highly elongated in the streamwise direction, is flanked on either side by pronounced negative correlation contours. As is expected from plots (a) and (b), the correlation maps compare well, despite the two orders of magnitude separating the experiments in terms of Reynolds number and boundary-layer thickness. Figure 7 shows a three-dimensional representation of the correlation contours of $R_{u u}$ for both the tower and the spanwise array deployed in the ASL. In keeping with Fig. 6, an assumed boundary-layer thickness of $\delta=60 \mathrm{~m}$ is used to non-dimensionalize the axes. The condition point is at s1 (shared with the second measurement station on the tower, t2). Viewed in this manner, it is clear that the streamwise/spanwise maps shown in Figs. $6 \mathrm{c}$ and $6 \mathrm{~d}$ have a large associated wall-normal extent (positive correlation extends beyond the top of the tower). The positive contours have a clear inclination in the streamwise direction, as previously noted for laboratory flows (e.g. Kovasznay et al. 1970; Brown and Thomas 1977; Christensen and Adrian 2001; Ganapathisubramani et al. 2005, etc.) and also in the ASL (Carper and Porté-Agel 2004; Marusic and Heuer 2007; Guala et al. 2011). The region of positive correlation is tall, thin and extremely persistent in the streamwise direction. 
The condition point for Fig. 7 is the shared sonic s1 (the second measurement station on the tower). If the condition point is relocated to a position in the spanwise array that is away from the tower we are able to investigate the two-point correlation coefficient over an altered spatial domain. This procedure is illustrated in Fig. 8a for the particular case of condition point s7 located $18 \mathrm{~m}$ to the west of the tower ( $\mathrm{s} 7$ is identified by a red circle in Fig. 8a). In this instance the $R_{u u}$ map from the vertical array gives the correlated behaviour on a streamwise / wall-normal plane $18 \mathrm{~m}$ to the side of the condition point. If this process is repeated for all ten possible condition points on the spanwise array (s1 - s10) it is possible to construct a volumetric view of the correlation behaviour. This process has been used previously by the authors (Marusic and Hutchins 2008; Hutchins et al. 2011) in laboratory measurements to map volumetric correlation information from simultaneously sampled orthogonal arrays / PIV planes. In the first instance, we will analyze the spanwise / wall-normal plane at $\Delta x=0$ from this correlation volume (such a 'cross-plane' is shown in Fig. 8a). Figure 8b shows the two-point correlation of the streamwise velocity fluctuation $R_{u u}$ on this cross-plane. This provides a view in cross-section of the tall and thin positive correlation regions, flanked in the spanwise direction by similarly sized anti-correlated regions. Figures 8c and $8 \mathrm{~d}$ show the correlation contours for the streamwise / spanwise fluctuations $\left(R_{u v}\right)$ and for the streamwise / wall-normal fluctuations $\left(R_{u w}\right)$ respectively. In these cases, the correlation contours are quite different. Comparing plots (b) and (d) reveals that the $R_{u w}$ contours are almost the opposite of $R_{u u}$. This implies that a large-scale negative $u$ fluctuation will be accompanied by a positive $w$ tendency (an upwash). Similarly, a high-speed $u$ region will be characterized by negative $w$ fluctuations. Both scenarios ('ejection' and 'sweep', or Q2 and Q4 events) are positive contributors to the Reynolds shear-stress. We can use these correlation maps to produce a Linear Stochastic Estimate (LSE) based on the occurrence of negative $u$ fluctuations (a low-speed event) at the condition point $\Delta y=0, z / \delta \approx 0.036$ (see Adrian and Moin 1988; Tomkins and Adrian 2003, for a description of this technique). For this simple detection event, the linear stochastic estimate is simply constructed from the two-point correlations $R_{u u}$, $R_{u v}$ and $R_{u w}$ calculated on unconditional data. With a negative $u$ fluctuation at the condition point (a condition vector of -1 ), the streamwise velocity vectors associated with this event are given by $-R_{u u}$, the spanwise vectors by $-R_{u v}$ and the wall-normal vectors by $-R_{u w}$ (this estimate was previously investigated on $45^{\circ}$ and $135^{\circ}$ inclined PIV planes for low Reynolds number laboratory flows by Hutchins et al. 2005). The resulting estimate is shown by the vector map in Fig. 8e. A large vortical motion accompanies the low-speed upwash event (at position $\Delta x=0, \Delta y=0 \& z / \delta=0.036$ ). Owing to the geometry of the measurement array ('L'-shaped arrangement), the esti- 

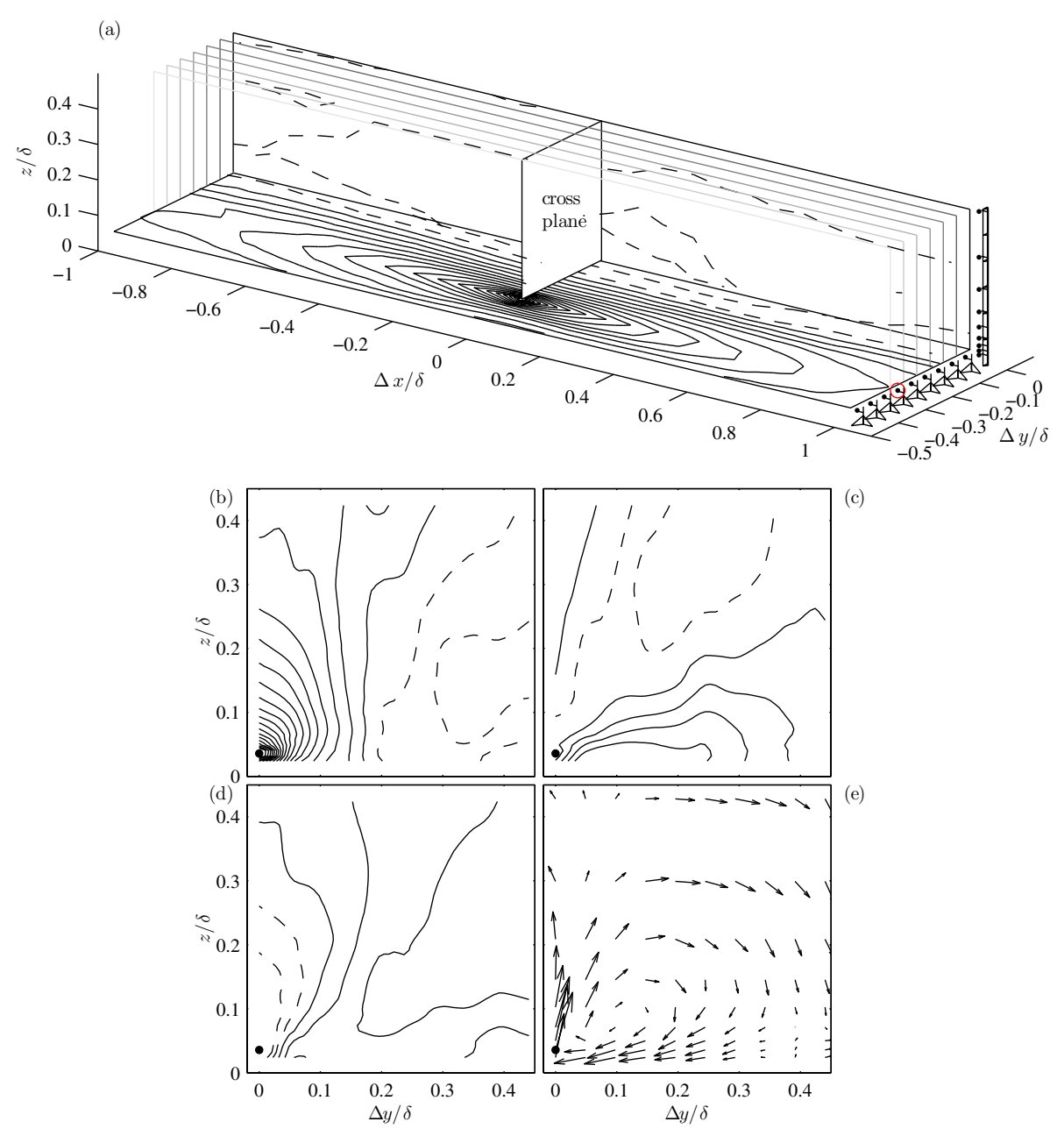

Fig. 8 (a) Mapping the correlation events over a volume. Iso-contours of $R_{u u}$ for both the spanwise array and the tower. The condition point at $\mathbf{s 7}$ is indicated by red circle. Symbols ' $\bullet$ indicate the position of sonic anemometers. (b) $R_{u u}$; (c) $R_{u v}$; (d) $R_{u w}$ calculated on the crossplane at $\Delta x=0 ;$ (e) linear stochastic estimate based on a negative $u$ event at the condition point $\Delta y=0, z / \delta \approx 0.036$. Contour levels for (a) and (b) are from $R_{u u}=-0.15$ to 0.95 in increments of 0.05 and from $R=-0.12$ to 0.12 in increments of 0.03 for (c) and (d). Solid lines show positive contours and dashed lines show negative levels.

mate is only obtained to the right of the condition point. However, since the boundary layer is two-dimensional, we can assume that the LSE results (and all preceding correlations) are symmetric in $y$ about the condition point (provided that they are suitably converged). In this way, it becomes clear that the low-speed event is accompanied by a counter-rotating vortex pair with upwash occurring between the two roll modes. A more detailed, volumetric view of this event is included as Fig. 9. This figure shows iso- 


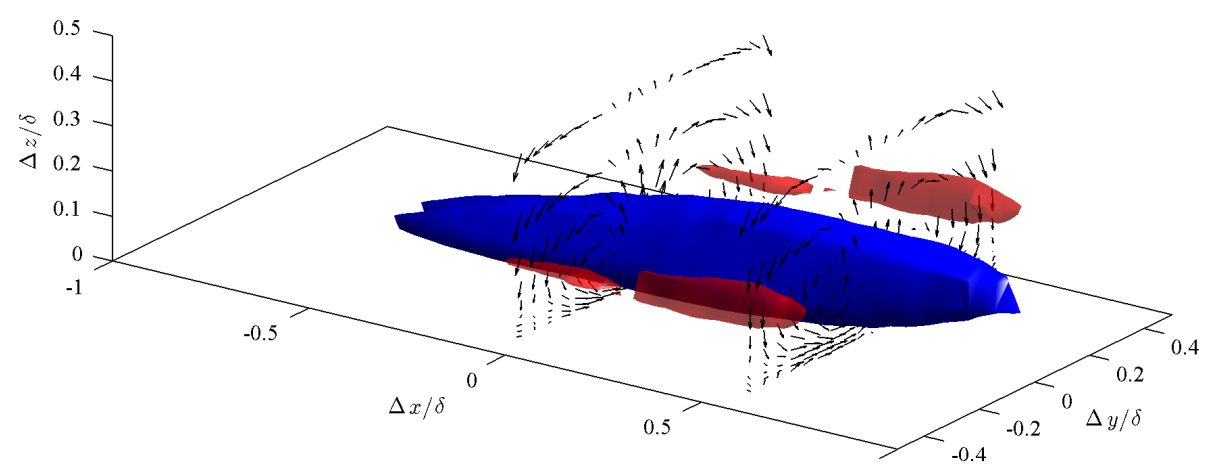

Fig. 9 The LSE event conditioned on a low-speed event (of magnitude -1 ) at $\Delta x=0$, $\Delta y=0, z / \delta \approx 0.036$; blue iso-surfaces show negative $u$ fluctuation $(u<-0.16)$, red iso-surfaces show positive $u$ fluctuation $(u>0.18) . v$ and $w$ vectors are shown on spanwise/wall-normal planes at $\Delta x / \delta=0$ and 0.5 .

surfaces of negative and positive streamwise velocity fluctuations associated with the LSE event conditioned on a negative $u$ fluctuation at $\Delta x=0, \Delta y=0, z / \delta=0.036$. The LSE has been reflected to produce this figure (in recognition of the assumed statistical two-dimensionality of the flow). Cross-plane vector fields included on this figure show the accompanying large-scale roll modes. This figure is built from unconditional twopoint correlations and as such the values of the iso-surfaces are somewhat misleading. The $R_{u u}$ correlation drops off rapidly from the condition point, and thus the smaller size of the red iso-surfaces reflects more their distance from this condition point rather than implying that positive $u$ fluctuations are smaller than negative. Regardless, the figure reinforces the notion of counter-rotating roll modes occurring between elongated high and low $u$ momentum regions.

The correlation maps shown previously, along with numerous previous studies (for example Kim and Adrian 1999; del Álamo et al. 2004; Hutchins and Marusic 2007a) indicate that the regions of negative $u$ fluctuation are large, especially in the streamwise direction where they are highly elongated. Thus, the LSE result incorporates a large degree of phase jitter, analogous to a spatial filter (since the condition criteria are met everywhere along the length of the elongated low-speed regions). This is in contrast to LSE results that are based on a spatially more compact condition event, such as swirl (Christensen and Adrian 2001; Hambleton et al. 2006). In these instances, the phase jitter is less acute, and the estimates better resolve the finer-scale structure of the flow. Such swirl-conditioned results indicate a fine-scale vortical structure existing about the low-speed regions in arrangements that are consistent with the hairpin packet paradigm (Zhou et al. 1999; Adrian et al. 2000b; Christensen and Adrian 2001; Ganapathisubra- 
mani et al. 2003; Tomkins and Adrian 2003). The absence of such finer features from the conditional view offered in Fig. 9 is a reflection only of the diffuse nature of the condition event. We would still expect these large-scale modes to be accompanied by fine-scale vortical motions (packets/clustering). It is, however, doubtful whether these fine-scale vortical motions can be correctly captured by our measurement array, since the sonic anemometers have a relatively poor spatial and temporal resolution. We believe that the presence of the large-scale roll modes in the two-point correlations are truly indicative of instantaneous flow features (and not merely a statistical artifact of the LSE technique). The spanwise velocity component due to these roll modes is clearly evident in $\S 5.2$, when we consider the instantaneous form of the large-scale structure. These large-scale roll modes have been previously observed in laboratory experiments and simulations of turbulent boundary layers. Marusic and Hutchins (2008) produced a volumetric LSE based on a low-speed event (using the low Reynolds number simultaneous orthogonal plane PIV data of Hambleton et al. 2006) finding evidence of largescale roll modes. In the cross-plane PIV study of Hutchins et al. (2005), instantaneous vector fields (Fig. 6d of Hutchins et al. 2005) contain evidence of large-scale diffuse counter-rotating motions flanking the tall low-speed regions. Such circulatory motions associated with the instantaneous large-scale structure have also been noted in recent DNS studies (Toh and Itano 2005). Jiménez and del Álamo (2004) and del Álamo et al. (2006) performed conditional analysis based on attached clusters of vortices, the results of which indicated the presence of large-scale elongated low-speed regions accompanied by a similar counter-rotating vortex pair to that shown in Fig. 9. In that study the authors have suggested that the vortex pair, "...is statistically important, because it takes part in the stirring of the mean profile that leads to the generation of the large scales of $u . "$ In a recent LES of turbulent channel flow, Chung and McKeon (2010) observed large-scale roll modes flanking superstructure/VLSM-type events. Certainly, the presence of these large-scale counter-rotating structures could hold vital clues to the formation mechanisms that are responsible for the very long $u$ structures so evident in the logarithmic region of turbulent boundary layers (they also provide a possible organising motion that could lead to the concatenation of packets/LSM as speculated by Guala et al. 2006; Adrian 2007; Dennis and Nickels 2011b).

In atmospheric flows, LES studies have found counter-rotating roll modes near the wall associated with conditionally sampled sweep and ejection events (Lin et al. 1996; Foster et al. 2006). Drobinski et al. (2004) suggested from LIDAR data that large-scale streaks are accompanied by spanwise converging / diverging motions consistent with roll modes. It should, however, be noted that these large-scale modes found in the ASL (often termed PBL rolls) are typically on a larger scale (when scaled with boundary- 
layer thickness $\delta$ ) than those observed here. It should also be pointed out that the large roll modes explained above are very different to the quasi-two-dimensional structures that occur in the convective mixed layer and are also called rolls (LeMone 1973; Khanna and Brasseur 1998; Young et al. 2002). Such convective rolls are buoyancy induced motions that form alternating regions of updrafts $(+w)$ and downdrafts $(-w)$. These motions extend throughout the atmospheric boundary-layer depth and are much bigger in size than the shear-induced roll modes discussed above. In considering why these large-scale vortical motions are so often overlooked in laboratory studies, it is useful to consider the vortex identification schemes that have become the standard tools in visualizing complex flows. Vortical structures are typically visualized by taking isosurfaces through the swirl (Adrian et al. 2000a), discriminant (Chong et al. 1998), $\lambda_{2}$ (Jeong and Hussain 1995) or variants thereof. The threshold values necessitated by such schemes will always favour more compact regions of tightly swirling motion and as such, the larger more diffuse circulatory motions associated with the large-scale structure in the logarithmic region will tend to be ignored. Only with spatial filtering (such as that achieved by two-point correlations) do these motions begin to manifest.

\subsection{Instantaneous structure}

We now look at the instantaneous velocity fluctuations in order to verify that the features suggested by the two-point correlation results in the previous section are present instantaneously in the surface layer. Figures 10d-10f respectively show fluctuations of $u, v$ and $u u w$ for streamwise / spanwise planes from the ASL. In Fig. 10c, instantaneous $u$ in the wall-normal plane is also shown, using data from the tower array. For comparison, Figs. 10a-10c show equivalent data from much lower Reynolds number laboratory PIV experiments (Hambleton et al. 2006). The PIV experiments were obtained at friction Reynolds number $R e_{\tau}=1100$ and the streamwise / spanwise PIV plane is located slightly further from the wall at $z / \delta \approx 0.1$. The Reynolds number for the ASL at SLTEST is three orders of magnitude higher, but the plane described by the spanwise array is comparable with $z / \delta \approx 0.036$ (both wall-parallel planes are in the logarithmic region). For the ASL data, the instantaneous time variation of $u$ over a period of 110 seconds is projected in space using Taylor's hypothesis to obtain the planar view. The mean velocity measured by the spanwise array provides the approximate convection velocity for this conversion. With $\delta=60 \mathrm{~m}$ for the ASL and $U=5.46 \mathrm{~m}$ $\mathrm{s}^{-1}$ (at $\left.z=2.14 \mathrm{~m}\right)$, this translates to a projected spatial domain of $10 \delta(\approx 600 \mathrm{~m})$ in the $x$-direction. A very long low-speed region (blue) in the spanwise plane is seen in

both Fig. 10a and 10d. The measured extent of the low-speed region for the PIV is limited to approximately $2 \delta$ due to the limited field of view of the camera. The extent 


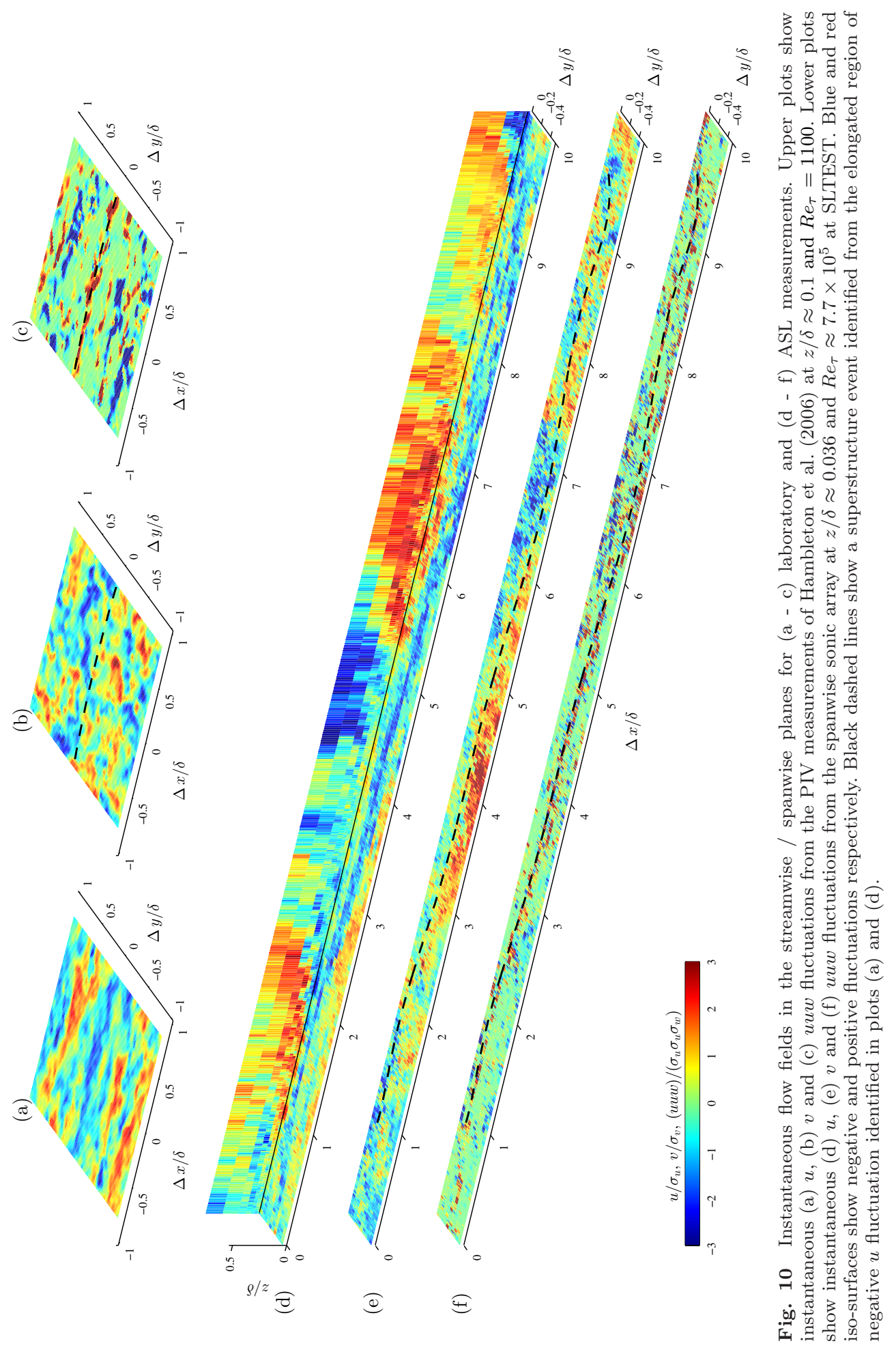



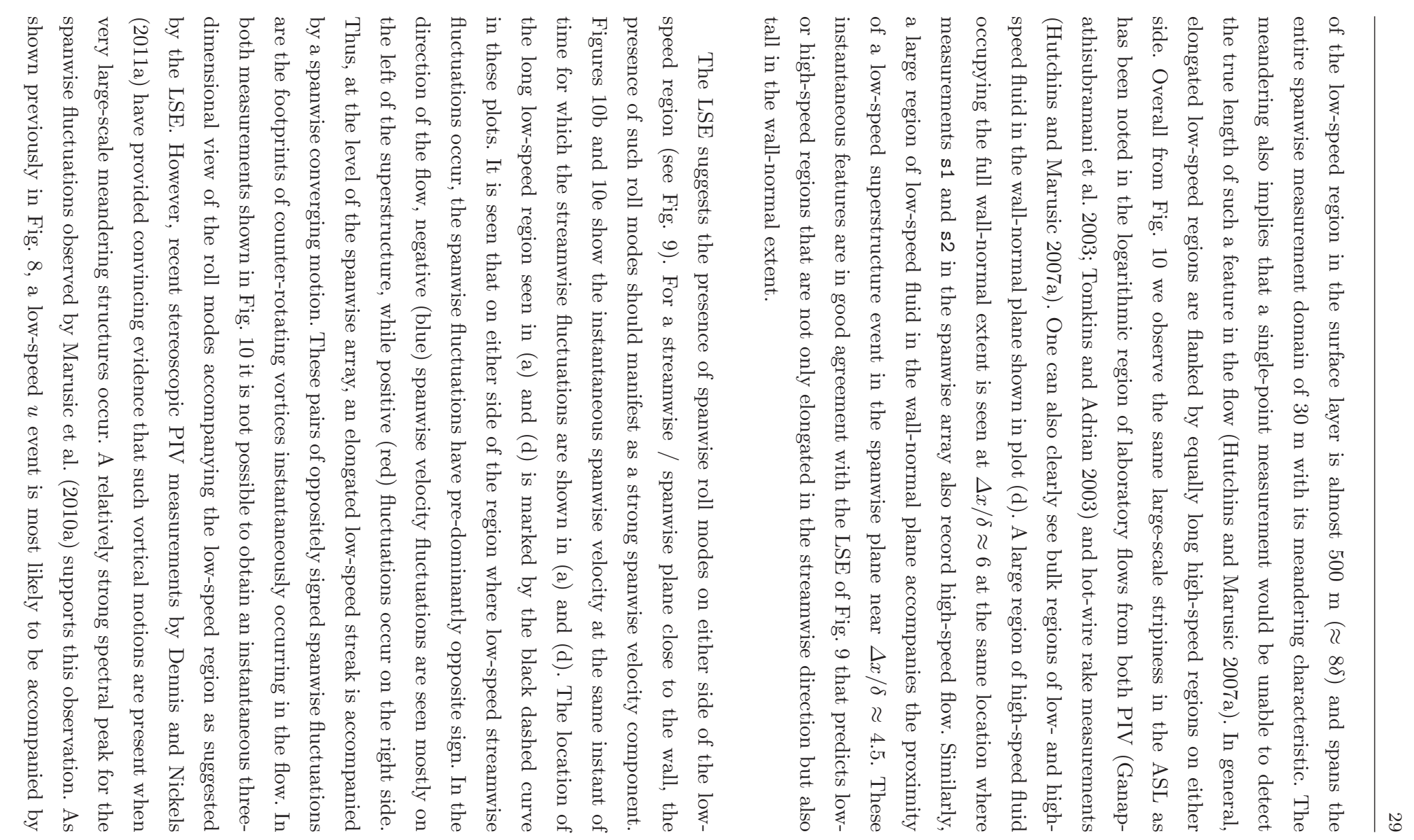
an upwash (thus low-speed $u$ events typically indicate the transport of low-momentum fluid away from the wall to the outer part of layer). Hence, the wall-normal fluctuations associated with the instantaneous superstructure/VLSM events shown in Fig. 10 are also of interest. Figures $10 \mathrm{c}$ and $10 \mathrm{f}$ show instantaneous fields of the triple component uuw for laboratory and ASL experiments respectively. The instantaneous Reynolds shear-stress $u w$ is the quantity of more interest than $w$ itself. Positive contributions to Reynolds shear-stress come from Q2 $(u<0 \& w>0)$ and Q4 $(u>0 \& w<0)$ events. The Q2 events result in a positive uuw and thereby the triple component uuw enables us to clearly distinguish from the Q4 events occurring in the high-speed regions that flank the superstructure ${ }^{4}$. The triple component can be thought of as the transport of streamwise normal stress due to wall-normal velocity fluctuations. The strong correlation between positive $w$ fluctuation and the elongated low-speed meandering regions (dashed black curve) is clearly observed in Figs 10c and 10f. This correlation is evident along the length of the superstructure unlike the short-lived ejection events detected from single-point measurements. Even though the individual wall-normal fluctuations are spatially quite compact close to the surface (Högström et al. 2002; Marusic et al. 2010a), it is clear that the superstructure event has a largescale organizing influence.

The laboratory turbulent boundary layer and ASL consistently exhibit instantaneous features that are representative of superstructures in the logarithmic region. The instantaneous features seen in Fig. 10 corroborate the inferences made from the twopoint correlation and LSE regarding the statistical footprint of large-scale motions; i.e. elongated and narrow high- and low-speed regions accompanied by counter-rotating roll modes. It should be further noted that the full streamwise and spanwise extent of these motions cannot be determined due to the limited size of the measurement array in the $y$-direction $(\approx 0.5 \delta)$, which is insufficient given the meandering tendency of these structures. However, the existence of these large structures in the ASL and at very large Reynolds numbers suggests their universality in wall-turbulence, albeit at present in zero-pressure-gradient turbulent boundary layers. The similarity of features observed in Fig. 10 between both the laboratory and atmospheric flow leads one to the discussion of whether the underlying wall-mechanisms are common in these flows (as has also been contemplated by Guala et al. 2011).

\footnotetext{
4 The triple component $u u w$ is also positive for Q1 events but these are not prominent in the flow and do not exhibit large-scale coherence.
} 


\section{Inclined vortices or Ramp events}

6.1 Statistical evidence: Two-point correlations

Next, we look for the signature of hairpin-like vortical structures in the flow. In the laboratory, hairpin vortices have been well-documented (for example Adrian et al. 2000b; Christensen and Adrian 2001; Ganapathisubramani et al. 2003, 2005; Lee and Sung 2011 , etc.) while in the ASL the evidence, though suggestive of such features, has been less conclusive (Hommema and Adrian 2003; Carper and Porté-Agel 2004; Morris et al. 2007). Typical signatures used to identify hairpin vortices are the occurrence of spanwise vortices in the streamwise / wall-normal plane representing the hairpin heads (e.g. Adrian et al. 2000b), and also counter-rotating vortex pairs in the streamwise / spanwise plane, representing the hairpin legs (Ganapathisubramani et al. 2003; Tomkins and Adrian 2003). Here we concentrate on the spanwise velocity component in the wall-normal plane which would be significant in the leg or arch of a typical hairpin structure. Figure 11 shows the two-point correlation of the $v$ fluctuations for both the laboratory turbulent boundary layer and the near-neutral ASL. Similar to the $R_{u u}$ result in Fig. 6, the overall features of the $R_{v v}$ correlation map are almost identical for laboratory and ASL measurements. Distinct regions of positive correlation surrounding the condition point give way to negatively correlated regions further from the wall, with a pronounced inclined separation between the two. A quasi-streamwise vortex inclined to the wall would have zero spanwise velocity in its core. Hence, statistically the zero correlation line of $R_{v v}$ could represent the core of an averaged vortex with spanwise fluctuations of opposite signs on either side. The zero correlation line in Figure 11 has a characteristic shape to it; i.e. it has a shallow linear inclination angle up to $\Delta x \approx 0$ and steep inclination for $\Delta x>0$. This implies that the vortical feature has two distinct orientations upstream and downstream of the condition point. Such an organization resembles quite closely the commonly accepted shape of a hairpin vortex, with shallow inclination for the leg and steeper angle for the arch (see Fig. 12c). The inclination angle of $25^{\circ}$ away from the wall is small compared to the original findings of Head and Bandyopadhyay (1981), who reported $45^{\circ}$, but in very good agreement with the $26.5^{\circ}$ found by Dennis and Nickels (2011a). Nevertheless, it would seem that inclined features, typical of hairpin-like vortices are present in the ASL and extend up to at least $0.5 \delta(30 \mathrm{~m})$ in the wall-normal direction. The instantaneous spanwise velocity field (on a streamwise / wall-normal plane) shown in Fig. 12 provides further evidence of these features. 

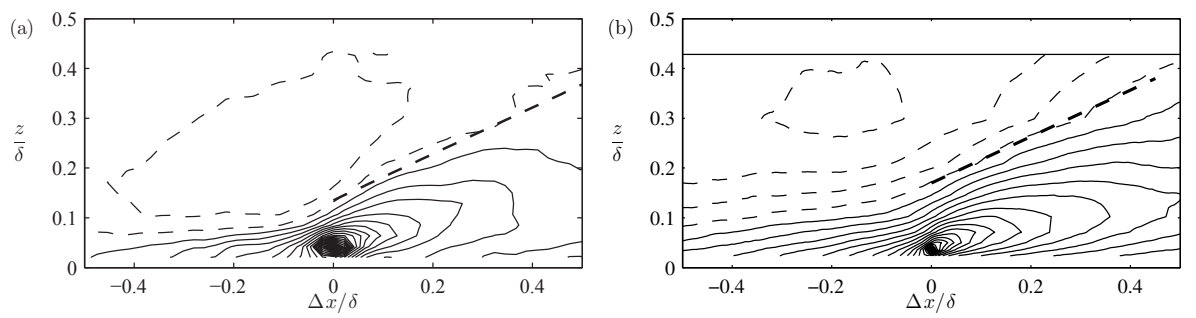

Fig. 11 Iso-contours of $R_{v v}$ in the streamwise/wall-normal plane for laboratory and ASL measurements. (a) PIV data of Hambleton et al. (2006) at $R e_{\tau}=1100$. The condition point is at $z / \delta \approx 0.042$. (b) ASL data from the sonic anemometer tower array at SLTEST. The condition point is at $z / \delta \approx 0.036$. The dashed straight lines in both figures indicate an inclination angle of $25^{\circ}$. In both (a) and (b) contour levels are from $R_{v v}=-0.5$ to 0.95 in increments of 0.05. Solid lines show positive contours and dashed lines show negative levels. ' $\bullet$ ' indicates the location of condition point. 


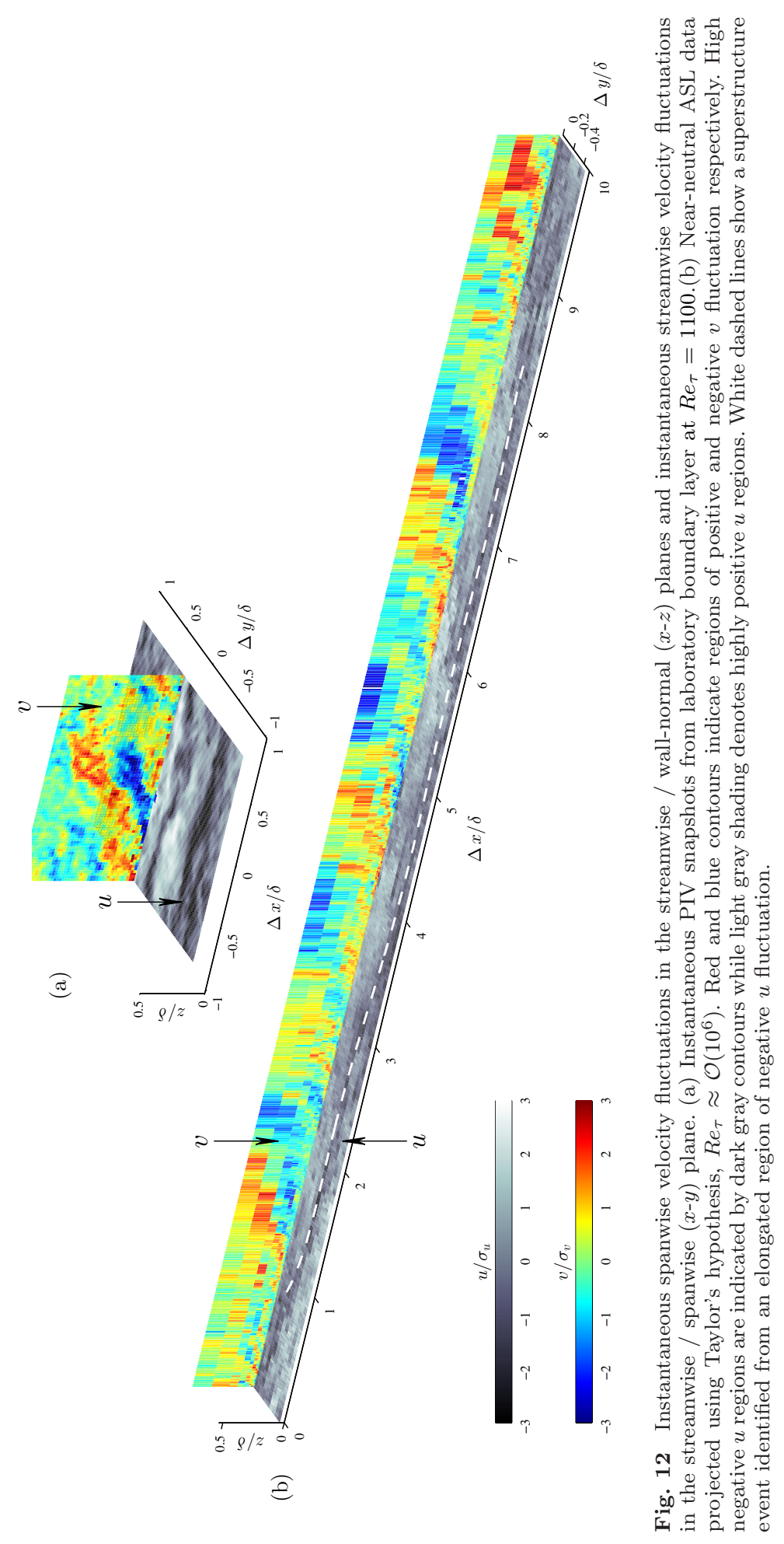




\subsection{Instantaneous structure}

The colour contours in Fig. 12a \& 12b show instantaneous spanwise velocity fluctuations on streamwise / wall-normal planes for both the PIV and sonic anemometer measurements. For the surface-layer data the spatial flow field is projected in $x$ using Taylor's hypothesis. The colour contours exhibit a distintive alternating pattern of inclined positive and negative $v$ fluctuations. These stripes of positive or negative $v$ stretch all the way from the wall to the core of the boundary layer. Multiple instances of this stripiness are evident, emphasizing that such features are abundantly present in the flow. The wall-normal extent of the flow field here is limited by the maximum height of the sonic anemometer array. Nevertheless we observe that these alternating inclined spanwise motions extend up to at least $0.5 \delta$. These instantaneous features agree remarkably well with the two-point correlation maps of Fig. 11. The inclination angle appears to be shallow near the wall becoming steeper as $z / \delta$ increases. Consistent with the similarity of $R_{v v}$ between the laboratory and atmospheric flows observed in Fig. 11, we note that the instantaneous spanwise velocity fields in Fig. 12 also have a strong resemblance. For both flows, the instantaneous spanwise velocity fluctuations are strongly suggestive of the flows that would be induced by the counter-rotating rolls suggested by the LSE in Fig. 6 and/or inclined vortices inferred from $R_{v v}$ in Fig. 11 . At this stage it would be premature to attribute each of the striped regions observed in the ASL to a single hairpin vortex. Rather, we should recognize that the ASL data represents a filtered view owing to the limited spatial and temporal resolution of the sonic anemometers and the logarithmic spacing of the sonics on the tower.

The grey-scale contours on Fig. 12 show the instantaneous streamwise velocity fluctuations on the streamwise / spanwise plane (for the same instant as the spanwise fluctuations shown by the colour contours). The streamwise velocity fluctuations show pronounced regions of elongated uniform momentum appearing as alternating stripes of positive and negative $u$ fluctuations in the streamwise / spanwise plane. For the PIV results, this coherence is only observed over an extent of about $1.7 \delta$ (owing to the limited field of view of the cameras). For the ASL, a very long meandering lowspeed region is observed in the wall-parallel plane. This superstructure (marked by the white dashed line in Fig. 12b) is at least $6 \delta$ long with flanking high-speed regions on either side. For both the laboratory and the ASL data, it is noted that there are distinctive inclined regions of alternating positive and negative spanwise velocity in the streamwise/wall-normal planes in locations where these planes are flanking the superstructure events. This observation is in support of the hairpin packet paradigm which proposes clusters of hairpin stacked together and residing over uniform streamwise momentum zones (Kim and Adrian 1999; Adrian 2007). 
(a)

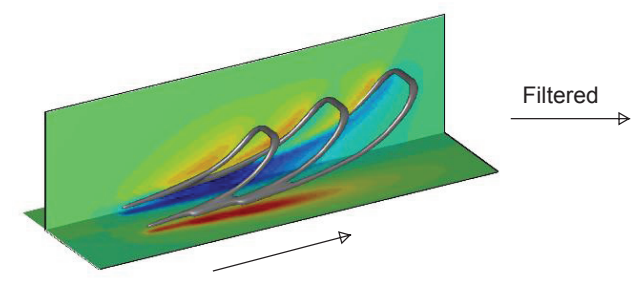

(b)

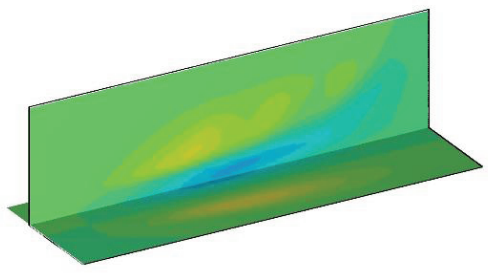

Fig. 13 Spanwise velocity fluctuations on a streamwise/wall-normal plane induced by a modelled packet of hairpin vortices. The velocity field is produced from a Biot-Savart calculation for vortex lines in the core of the hairpin shapes shown. In both (a) and (b) blue and red iso-surfaces imply negative and positive $v$ fluctuations respectively. (b) shows box-filtered $v$ velocity field.

To qualitatively examine the occurrence of inclined spanwise velocity regions due to hairpin vortices, Fig. 13a-13b shows the synthetic induced velocity field due to an idealized modelled hairpin packet. Vortex line segments are arranged into hairpin shapes, which are then repeated to generate the packet feature. The induced velocity field due to these vortex lines, together with their image vortices in the wall (Perry and Marusic 1995) is calculated using the Biot-Savart law. Contours of spanwise velocity in the streamwise / spanwise and streamwise / wall-normal planes are shown. The streamwise / wall-normal plane is chosen such that it cuts through the inclined legs of the hairpin packet. It is found that alternating regions of positive and negative spanwise velocity are formed in the wall-normal plane which look qualitatively similar to the inclined regions observed in Fig. 12. The figure also indicates that it is likely that the stripiness observed in the instantaneous field is due to a packet of vortices moving together rather than an individual hairpin vortex. The calculated velocity field is thereafter box-filtered and contours for the resulting velocity field are shown in Fig. 13b. In a filtered view the packet appears as a single inclined negative and positive spanwise velocity region, at a much larger physical scale in the streamwise/wall-normal plane resembling remarkably well the large-scale spanwise velocity fluctuations observed in Fig. 12. As mentioned previously, the measurements by PIV and sonic anemometry both suffer from inherent spatial and temporal averaging. Owing to this, it is likely that the spanwise velocity map in Fig. 12 represents a filtered view that can only reveal the large-scale induced velocity fluctuations due to clusters or packets of vortical structures, while failing to resolve individual hairpin vortices. However, in spite of this resolution issue, the similarity in statistics and instantaneous features of spanwise velocity fluctuations might suggest that hairpin-like vortices or packets are present, or at least are consistent with experimental observations, over a wide range of physical scales $\left[\mathcal{O}\left(10^{-2} \mathrm{~m}\right)-\mathcal{O}(10 \mathrm{~m})\right]$ 
and Reynolds numbers $\left[\mathcal{O}\left(10^{3}\right)-\mathcal{O}\left(10^{6}\right)\right]$. This observation is in contrast to the suggestion by Guala et al. (2010), that hairpin packets in the ASL may be confined to the near-wall layer of $z^{+}=\mathcal{O}\left(10^{3}\right)$. For the present case the lowest measurement station on the tower has $z^{+}>10^{4}$.

Finally, we examine the wall-normal velocity component and its coupling with the streamwise fluctuations in the streamwise / wall-normal plane. The inclined structure of $R_{u u}$ in Fig. 7 indicates the presence of ramp-like features in the flow. The hairpin packet model proposes organization of hairpin vortices in a ramp-like orientation with low-momentum fluid trapped between their arches and legs and inducing organized contributions to Reynolds shear-stress. Figure 14 shows contours of instantaneous streamwise velocity fluctuations $u$ and the turbulent transport term $u u w$ for the laboratory turbulent boundary layer and the ASL. Ganapathisubramani et al. (2003) and Lee and Sung (2011) have found that more than $25 \%$ of the total Reynolds shear-stress is associated with hairpin vortices (Guala et al. 2006, suggest the contribution could be as large as 50\%). Hence, an examination of high Reynolds shear-stress regions, particularly Q2 and Q4 events indirectly point to the signature of such vortices. Just as in Fig. 10, we here focus on the Q2 event (ejection) only. Figures 14a and 14c show large regions of low-momentum fluid inclined away from the wall (dashed lines are at $14^{\circ}$ ) indicating a ramp-like organization of high-shear zones. In laboratory flows, ramp-like structures are found at an angle of approximately $10^{\circ}-13^{\circ}$ (Dennis and Nickels 2011b; Christensen and Adrian 2001), while in the ASL the reported angle varies between $3^{\circ}-35^{\circ}$ (Hommema and Adrian 2003) depending on the height. It is seen that high Reynolds shear-stress regions occur at the same location in Figs. 14b and 14d corresponding to the low momentum regions in (a) and (c). Hence, a significant transport of momentum occurs along these inclined structures, transporting the near-wall fluid to the outer layer. Although it is not possible to identify the underlying mechanism creating these structures, it is clear that the characteristics of hairpin packets in laboratory flows (i.e. ramp-like organization associated with high Reynolds shear-stress) are also convincingly found in the atmospheric surface layer. Also low-momentum zones in the ramp-like features extend all the way up to $0.7 \delta$ for the laboratory turbulent boundary layers. The present ASL measurements are limited to $26 \mathrm{~m}$, however, given the similarity of features in Fig. 14a \& 14c the existence of ramp-like features up to $50 \mathrm{~m}$ in the ASL should not be surprising. To summarize this section, we find instantaneous large-scale flow structures on the $\mathcal{O}(2 \delta)$ streamwise length that are fully consistent with hairpin packet model of Adrian et al. (2000b). However, at this stage the limited resolution (both spatial and temporal) prevents the conclusive identification of individual vortex cores. 


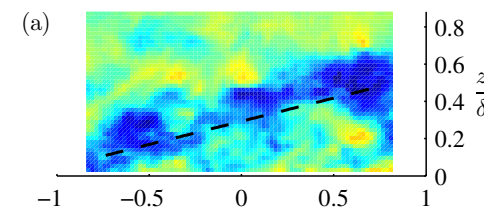

(b)

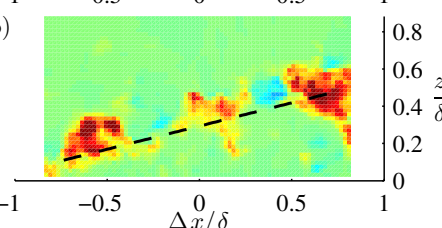

$u / \sigma_{u},(u u w) /\left(\sigma_{u} \sigma_{u} \sigma_{w}\right)$
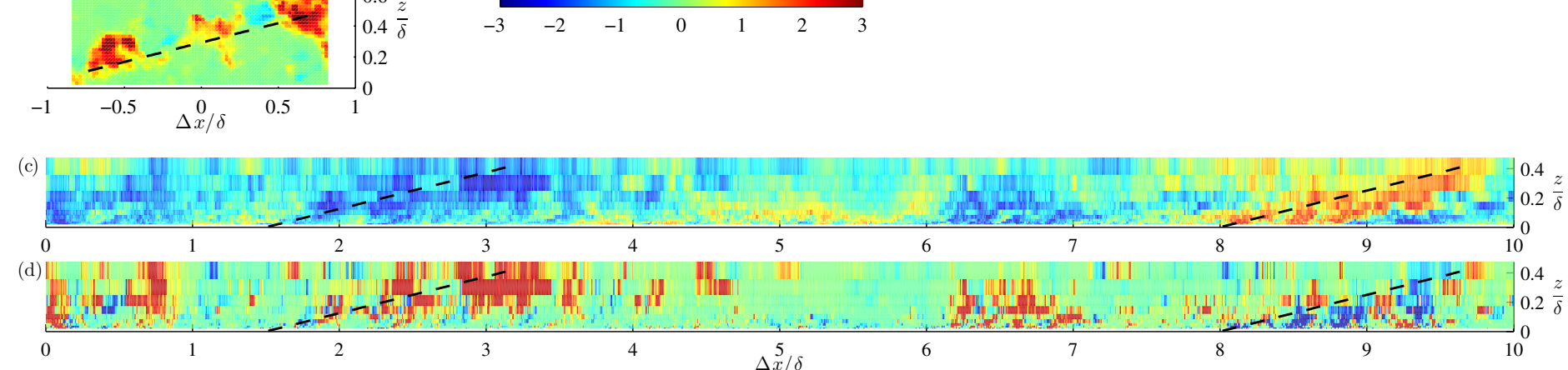

Fig. 14 Streamwise / wall-normal planes showing the instantaneous flow fields for both (a, b) laboratory and (c, d) ASL measurements. (a) and (b) are instantaneous $u$ and $u u w$, respectively from PIV of Hambleton et al. (2006) at $R e_{\tau}=1100$. (c) and (d) are instantaneous $u$ and $u u w$, respectively from the tower sonic array at SLTEST. Blue and red iso-surfaces show regions of negative and positive fluctuation respectively. Dashed lines indicate an inclination angle of $14^{\circ}$. 


\section{Conclusions}

The mean statistics and large-scale coherent structures are compared between laboratory turbulent boundary layers and the near-neutral atmospheric surface layer developing over the uniquely flat and smooth terrain of the Utah salt flats. By all measures tested, and within the stated measurement uncertainty, it is concluded that the ASL under near-neutral conditions behaves precisely as a canonical flat plate turbulent boundary layer in the logarithmic region (provided one accounts for the very high Reynolds numbers of the ASL). Although the measurement of secondary quantities such as $U_{\tau}$ and $\delta$ are challenging in the ASL, the theoretical similarity of $U^{+}$(logarithmic behaviour) and $-\overline{u w}$ is observed to hold well between the ASL and the laboratory turbulent boundary layer.

At a structural level, the large-scale coherence in the logarithmic region of both flows has been compared in detail. Analysis of two-point correlation maps from the ASL indicates the presence of large-scale structures that are entirely consistent with the superstructures (Hutchins and Marusic 2007a) or VLSM (Kim and Adrian 1999) observed previously in laboratory flows. When scaled with the boundary-layer thickness, the two-point correlation maps from the ASL are virtually indistinguishable from those measured in laboratory turbulent boundary layers. Instantaneous flow fields provide further evidence of superstructure-type events, indicating highly elongated low/high speed regions, that again match those observed in lower Reynolds number flows, both

in scale and meandering behaviour. These features scale with the boundary-layer thickness $\delta$ and have been found to commonly exceed $10 \delta$ in length. In the ASL, such features are of the kilometre scale, and can take several minutes to advect past a stationary measurement array. The conditional flow fields have been further analyzed using linear stochastic estimates, which reveal the presence of counter-rotating roll modes on either side of the superstructure events. The large-scale low-momentum region of the superstructure is associated with upwash between the two rolls. These roll modes are again consistent with the wealth of observations from lower Reynolds number laboratory flows, where they are found to accompany the superstructure events (Jiménez and del Álamo 2004; Toh and Itano 2005; del Álamo et al. 2006; Hutchins and Marusic 2007b; Marusic and Hutchins 2008; Chung and McKeon 2010). The instantaneous flow fields also support the presence of these roll modes, evident as very long regions of converging spanwise velocity that straddle the low-speed streamwise motion. Owing to the fact that these features scale with the boundary-layer thickness, the approximate diameter of the roll modes tend to be very large in the ASL, $\mathcal{O}(0.5 \delta \approx 30 \mathrm{~m})$.

Due to the limited spatial and temporal resolutions of the L-shaped measurement array used for the ASL measurements, it is not possible to ascertain the presence 
of individual hairpin vortices by conventional interrogation of the instantaneous flow fields. Instead, the spanwise velocity component in streamwise/wall-normal planes is examined for signs of hairpin vortex signatures. To our knowledge, this is the first time that such data have been analyzed for this purpose. In both the laboratory and ASL, the distinctive form of the $R_{v v}$ correlation map exhibits the signature of an averaged vortex that has a shallow inclination angle upstream of the condition point, and a steeper angle downstream. The instantaneous flow fields again support this observation with alternating inclined regions of positive and negative spanwise velocity observed throughout the streamwise-wall-normal planes. These same features are observed in the laboratory turbulent boundary layer and in the ASL. In the surface layer such features extend to considerable heights from the wall (commonly extending beyond $30 \mathrm{~m}$, the limit of the wall-normal array). This physical length scale is indicative of the enormous scale separation that exists between the largest and smallest vortical motions in the ASL. It is likely that the features observed here are packets or clusters of many vortices, rather than individual vortical events. However, the remarkable similarity between these features observed in the ASL and those observed in low Reynolds number laboratory PIV experiments suggest that the vortical features in the logarithmic regions of both flows share a strong commonality.

Acknowledgements The authors gratefully acknowledge support from the National Science Foundation, the David and Lucile Packard Foundation, the Office of Naval Research and the Australian Research Council. The SLTEST measurements in 2005 were a collaborative effort involving a large team from several universities. Specifically we acknowledge the support of staff at the Dugway Proving Ground (in particular Donny Storwold), Ed Swiatek from Campbell Scientific, Keith McNaughton and Rob Clement of the University of Edinburgh and Todd Novak of the University of Minnesota.

\section{References}

Abe H, Kawamura H, Choi H (2004) Very large-scale structures and their effects on the wall shear-stress fluctuations in a turbulent channel flow up to $R e_{\tau}=640$. J Fluids Eng $126: 835-843$

Adrian RJ (2007) Hairpin vortex organization in wall turbulence. Phys Fluids 19(04):1301

Adrian RJ, Moin P (1988) Stochastic estimation of organized turbulent structure: homogeneous shear flow. J Fluid Mech 190:531-559

Adrian RJ, Christensen KT, Lui ZC (2000a) Analysis and interpretation of instantaneous turbulent velocity fields. Exp Fluids 29:275-290

Adrian RJ, Meinhart CD, Tomkins CD (2000b) Vortex organization in the outer region of the turbulent boundary layer. J Fluid Mech 422:1-54

del Álamo JC, Jiménez J, Zandonade P, Moser RD (2004) Scaling of the energy spectra of turbulent channels. J Fluid Mech 500:135-144

del Álamo JC, Jiménez J, Zandonade P, Moser RD (2006) Self-similar vortex clusters in the logarithmic region. J Fluid Mech 561:329-358

Bandyopadhyay PR, Hussain AKMF (1984) The coupling between scales in shear flows. Phys Fluids 27(9):2221-2228

Boppe RS, Neu WL (1995) Quasi-coherent structures in the marine atmospheric surface layer. J Geophys Res 100(C10):20635-20648 
Brown GL, Thomas ASW (1977) Large structure in a turbulent boundary layer. Phys Fluids 20(10):243-252

Carlotti P (2002) Two-point properties of atmospheric turbulence very close to the ground: Comparison of a high resolution LES with theoretical models. Boundary-Layer Meteorol 104(3):381-410

Carper MA, Porté-Agel F (2004) The role of coherent structures in subfilter-scale dissipation of turbulence measured in the atmospheric surface layer. J Turb 5(40)

Chauhan KA (2007) Study of canonical wall-bounded turbulent flows. PhD thesis, Illinois Institute of Technology, Chicago

Chong MS, Soria J, Perry AE, Chacin J, Cantwell BJ, Na Y (1998) Turbulence structures of wall-bounded shear flows found using DNS data. J Fluid Mech 357:225-247

Christensen KT, Adrian RJ (2001) Statistical evidence of hairpin vortex packets in wall turbulence. J Fluid Mech 431:433-443

Chung D, McKeon BJ (2010) Large-eddy simulation of large-scale structures in long channel flow. J Atmos Sci 661:341-364

DeGraaff DB, Eaton JK (2000) Reynolds number scaling of the flat-plate turbulent boundary layer. J Fluid Mech 422:319-346

Dennis DJC, Nickels TB (2008) On the limitations of Taylor's hypothesis in constructing long structures in a turbulent boundary layer. J Fluid Mech 614:197-206

Dennis DJC, Nickels TB (2011a) Experimental measurement of large-scale three-dimensional structures in a turbulent boundary layer. Part 1. Vortex packets. J Fluid Mech 673:180-217

Dennis DJC, Nickels TB (2011b) Experimental measurement of large-scale three-dimensional structures in a turbulent boundary layer. Part 2. Long structures. J Fluid Mech 673:218 244

Drobinski P, Foster RC (2003) On the origin of near-surface streaks in the neutrally-stratified planetary boundary layer. Boundary-Layer Meteorol 108(2):247-256

Drobinski P, Carlotti P, Newsom R, Banta RM, Foster RC, Redelsperger JL (2004) The structure of the near-neutral atmospheric surface layer. J Atmos Sci 61(6):699-714

Foster RC, Vianey F, Drobinski P, Carlotti P (2006) Near-surface coherent structures and the vertical momentum flux in a large-eddy simulation of the neutrally-stratified boundary layer. Boundary-Layer Meteorol 120(2):229-255

Ganapathisubramani B, Longmire EK, Marusic I (2003) Characteristics of vortex packets in turbulent boundary layers. J Fluid Mech 478:35-46

Ganapathisubramani B, Hutchins N, Hambleton WT, Longmire EK, Marusic I (2005) Investigation of large-scale coherence in a turbulent boundary layer using two-point correlations. J Fluid Mech 524:57-80

Guala M, Hommema S, Adrian R (2006) Large-scale and very-large-scale motions in turbulent pipe flow. J Fluid Mech 554:521-542

Guala M, Metzger M, McKeon BJ (2010) Intermittency in the atmospheric surface layer: Unresolved or slowly varying? Physica D 239:1251-1257

Guala M, Metzger M, McKeon BJ (2011) Interactions within the turbulent boundary layer at high Reynolds number. J Fluid Mech 666:573-604

Hambleton W, Hutchins N, Marusic I (2006) Simultaneous orthogonal plane PIV measurements in a turbulent boundary layer. J Fluid Mech 560:53-64

Head MR, Bandyopadhyay P (1981) New aspects of turbulent boundary-layer structure. J Fluid Mech 107:297-337

Herpin S, Stanislas M, Soria J (2010) The organization of near-wall turbulence: a comparison between boundary layer SPIV data and channel flow DNS data. J Turbulence 11(47):1-30

Högström U, Bergström H (1996) Organized turbulence structures in the near-neutral atmospheric surface layer. J Atmos Sci 53(17):2452-2464

Högström U, Hunt JCR, Smedman AS (2002) Theory and measurements for turbulence spectra and variances in the atmospheric neutral surface layer. Boundary-Layer Meteorol 103(1):101-124

Hommema S, Adrian RJ (2003) Packet structure of surface eddies in the atmospheric boundary layer. Boundary-Layer Meteorol 106:147 - 170

Hunt JCR, Carlotti P (2001) Statistical structure at the wall of the high Reynolds number turbulent boundary layer. Flow Turb Combus 66:453-475

Hunt JCR, Morrison JF (2001) Eddy structure in turbulent boundary layers. Eur J Mech B Fluids 19:673-694 
Hutchins N, Marusic I (2007a) Evidence of very long meandering streamwise structures in the logarithmic region of turbulent boundary layers. J Fluid Mech 579:1-28

Hutchins N, Marusic I (2007b) Large-scale influences in near-wall turbulence. Phil Trans Royal Soc Lond A 365:647-664

Hutchins N, Hambleton WT, Marusic I (2005) Inclined cross-stream stereo particle image velocimetry measurements in turbulent boundary layers. J Fluid Mech 541:21-54

Hutchins N, Nickels TB, Marusic I, Chong MS (2009) Hot-wire spatial resolution issues in wall-bounded turbulence. J Fluid Mech 635:103-136

Hutchins N, Monty J, Ganapathisubramani B, Ng H, Marusic I (2011) Three-dimensional conditional structure of a high-Reynolds-number turbulent boundary layer. J Fluid Mech 673:255-285

Inagaki A, Kanda M (2010) Organized structure of active turbulence over an array of cubes within the logarithmic layer of atmospheric flow. Boundary-Layer Meteorol 135(2):209-228

Jeong J, Hussain F (1995) On the identification of a vortex. J Fluid Mech 285:69-94

Jiménez J (2004) Turbulent flows over rough walls. Annu Rev Fluid Mech 36:173-196

Jiménez J, del Álamo JC (2004) Computing turbulent channels at experimental Reynolds numbers. In: Proc. 15th Australasian Fluid Mech. Conf, URL http://www.aeromech.usyd.edu.au/15afmc/index.html, Sydney

Jiménez J, Pinelli A (1999) The autonomous cycle of near-wall turbulence. J Fluid Mech 389:335-359

Kaimal JC, Finnigan JJ (1994) Atmospheric Boundary Layer Flows: Their Structure and Measurement. Oxford University Press, New York

Khanna S, Brasseur J (1998) Three-dimensional buoyancy- and shear-induced local structure of the atmospheric boundary layer. J Atmos Sci 55(5):710-743

Kholmyansky M, Moriconi L, Tsinober A (2007) Large-scale intermittency in the atmospheric boundary layer. Phys Rev E 76(2):026,307

Kim KC, Adrian R (1999) Very large-scale motion in the outer layer. Phys Fluids 11:417-422

Klewicki JC (2010) Reynolds number dependence, scaling, and dynamics of turbulent boundary layers. J Fluids Eng 132(9):094,001

Klewicki JC, Metzger MM, Kelner E, Thurlow EM (1995) Viscous sublayer flow visualizations at $R_{\theta} \cong 1500000$. Phys Fluids 7:857-863

Kline SJ, Reynolds WC, Schraub FA, Rundstadler PW (1967) The structure of turbulent boundary layers. J Fluid Mech 30:741-773

Knobloch K, Fernholz HH (2002) Statistics, correlations, and scaling in a turbulent boundary layer at $\operatorname{Re}_{\delta_{2}} \leq 1.15 \times 10^{5}$. In: Smits AJ (ed) IUTAM symposium on Reynolds number scaling in turbulent flow, Princeton University, USA, Springer, pp 11-16

Kovasznay LSG, Kibens V, Blackwelder RF (1970) Large-scale motion in the intermittent region of a turbulent boundary layer. J Fluid Mech 41:283-326

Kulandaivelu V, Marusic I (2010) Evolution of zero pressure gradient turbulent boundary layers. In: Proc. 17th Australasian Fluid Mech. Conf., Paper no. 196, Auckland

Kunkel GJ (2003) An experimental study of the high Reynolds number turbulent boundary layer. PhD thesis, University of Minnesota, USA

Kunkel GJ, Marusic I (2006) Study of the near-wall-turbulent region of the high-Reynoldsnumber boundary layer using an atmospheric flow. J Fluid Mech 548:375-402

Lee JH, Sung HJ (2011) Very-large-scale motions in a turbulent boundary layer. J Fluid Mech $673: 80-120$

LeMone MA (1973) The structure and dynamics of horizontal roll vortices in the planetary boundary layer. J Atmos Sci 30(6):1077-1091

Lin CL, McWilliams JC, Moeng CH, Sullivan PP (1996) Coherent structures and dynamics in a neutrally stratified planetary boundary layer flow. Phys Fluids 8(10):2626

Lu H, Porté Agel F (2010) A modulated gradient model for large-eddy simulation: Application to a natural atmospheric boundary layer. Phys Fluids 22(1):015109

Marusic I, Heuer W (2007) Reynolds number invariance of the structure inclination angle in wall turbulence. Phys Rev Lett 99(114504)

Marusic I, Hutchins N (2008) Study of the log-layer structure in wall turbulence over a very large range of Reynolds number. Flow Turb Combus 81:115-130

Marusic I, Kunkel GJ (2003) Streamwise turbulence intensity formulation for flat-plate boundary layers. Phys Fluids 15:2461-2464

Marusic I, Hutchins N, Mathis R (2010a) High Reynolds number effects in wall-turbulence. J Heat Fluid Flow 31:418-428 
Marusic I, McKeon BJ, Monkewitz PA, Nagib HM, Smits AJ, Sreenivasan KR (2010b) Wallbounded turbulent flows at high Reynolds numbers: Recent advances and key issues. Phys Fluids 22:065103

Marusic I, Mathis R, Hutchins N (2003) Predictive Model for Wall-Bounded Turbulent Flow. Science 329:193-196

Mathis R, Hutchins N, Marusic I (2009) Large-scale amplitude modulation of the small-scale structures of turbulent boundary layers. J Fluid Mech 628:311-337

Mclean IR (1990) The near-wall eddy structure in an equilibrium turbulent boundary layer. $\mathrm{PhD}$ thesis, University of Southern California, USA

Metzger MM, Klewicki JC (2001) A comparative study of near-wall turbulence in high and low Reynolds number boundary layers. Phys Fluids 13:692-701

Metzger MM, Klewicki JC, Bradshaw KL, Sadr R (2001) Scaling the near-wall axial turbulent stress in the zero pressure gradient boundary layer. Phys Fluids 13:1819-1821

Moeng CH, Sullivan PP (1994) A comparison of shear- and buoyancy driven planetary boundary layer flows. J Atmos Sci 51:999

Monty J, Stewart J, Williams R, Chong M (2007) Large-scale features in turbulent pipe and channel flows. J Fluid Mech 589:147-156

Morris SC, Stolpa SR, Slaboch PE, Klewicki JC (2007) Near-surface particle image velocimetry measurements in a transitionally rough-wall atmospheric boundary layer. J Fluid Mech 580:319-338

Morrison JF (2007) The interaction between inner and outer regions of turbulent wall-bounded flow. Phil Trans Royal Soc Lond A 365:683-698

Nagib HM, Chauhan KA (2008) Variations of von Kármán coefficient in canonical flows. Phys Fluids 20:101518

Naguib AM, Gravante S, Wark CE (1996) Extraction of turbulent wall-pressure time-series using an optimal filtering scheme. Exp Fluids 22:14-22

Nakagawa H, Nezu I (1981) Structure of space-time correlations of bursting phenomena in an open-channel flow. J Fluid Mech 104:1-43

Newsom R, Calhoun R, Ligon D, Allwine J (2008) Linearly organised turbulence structures observed over a suburban are by dual-doppler LIDAR. Boundary-Layer Meteorol. 127:111130

Österlund JM, Johansson AV, Nagib HM, Hites MH (2000) A note on the overlap region in turbulent boundary layers. Phys Fluids 12(1):1-4

Panton RL (2001) Overview of the self-sustaining mechanisms of wall turbulence. Prog Aero Sci 37:341-383

Perry AE, Marusic I (1995) A wall wake model for the turbulent structure of boundary layers. Part 1. Extension of the attached eddy hypothesis. J Fluid Mech 298:361-388

Phong-Anant D, Antonia RA, Chambers AJ, Rajagopalan S (1980) Features of the organized motion in the atmospheric surface layer. J Geophys Res 85(C1):424-432

Piomelli U, Ferziger J, Moin P, Kim J (1989) New approximate boundary conditions for large eddy simulations of wall-bounded flows. Phys Fluids A 1(6):1061-1068

Robinson SK (1991) Coherent motions in turbulent boundary layers. Annu Rev Fluid Mech 23:601-639

Schoppa W, Hussain F (2002) Coherent structure generation in near-wall turbulence. J Fluid Mech 453:57-108

Smith CR, Metzler SP (1983) The characteristics of low-speed streaks in the near-wall region of a turbulent boundary layer. J Fluid Mech 129:27-54

Smits AJ, McKeon BJ, Marusic I (2011) High-Reynolds number wall turbulence. Annu Rev Fluid Mech 43:353-375

Spalart PR (1988) Direct numerical simulation of a turbulent boundary layer upto $R_{\theta}=1410$. J Fluid Mech 187:61-98

Sreenivasan KR (1985) On the fine scale intermittency of turbulence. J Fluid Mech 151:81-103

Theodorsen T (1952) Mechanism of turbulence. In: Proc. Second Midwestern Conference on Fluid Mechanics, Mar. 17-19, Ohio State Univesity, Columbus, Ohio

Toh S, Itano T (2005) Interaction between a large-scale structure and near-wall structures in channel flow. J Fluid Mech 524:249-262

Tomkins CD, Adrian RJ (2003) Spanwise structure and scale growth in turbulent boundary layers. J Fluid Mech 490:37-74

Townsend AA (1956) The Structure of Turbulent Shear Flow. Cambridge University Press 
Wark CE, Naguib AM, Robinson SK (1991) Scaling of spanwise length scales in a turbulent boundary layer. AIAA-paper 91-0235, 29th Aerospace Sciences Meeting, Nevada.

Wilczak JM, Tillman JE (1980) The three-dimensional structure of convection in the atmospheric surface layer. J Atmos Sci 37(11):2424-2433

Wu X, Moin P (2009) Direct numerical simulation of turbulence in a nominally zero-pressuregradient flat-plate boundary layer. J Fluid Mech 630:5-41

Wu Y, Christensen KT (2006) Population trends of spanwise vortices in wall turbulence. J Fluid Mech 568:55-76

Wyngaard JC (1992) Atmospheric turbulence. Annu Rev Fluid Mech 24:205-233

Young GS, Kristovich DAR, Hjelmfelt MR, Foster RC (2002) Rolls, streets, waves, and more: A review of quasi-two-dimensional structures in the atmospheric boundary layer. Bull Amer Meteorol Soc 83(7):997-1001

Zhou J, Adrian RJ, Balachandar S, Kendall TM (1999) Mechanisms for generating coherent packets of hairpin vortices in channel flow. J Fluid Mech 387:353-396 


\section{University Library}

\section{- M M I N E R VA A gateway to Melbourne's research publications}

Minerva Access is the Institutional Repository of The University of Melbourne

\section{Author/s:}

Hutchins, N;Chauhan, K;Marusic, I;Monty, J;Klewicki, J

Title:

Towards Reconciling the Large-Scale Structure of Turbulent Boundary Layers in the Atmosphere and Laboratory

\section{Date:}

2012-11-01

\section{Citation:}

Hutchins, N., Chauhan, K., Marusic, I., Monty, J. \& Klewicki, J. (2012). Towards Reconciling the Large-Scale Structure of Turbulent Boundary Layers in the Atmosphere and Laboratory. BOUNDARY-LAYER METEOROLOGY, 145 (2), pp.273-306. https://doi.org/10.1007/ s10546-012-9735-4.

Persistent Link:

http://hdl.handle.net/11343/282579 